\title{
Iterative positive solutions to a coupled fractional differential system with the multistrip and multipoint mixed boundary conditions
}

\author{
Xiaodi Zhao', Yuehan Liu and Huihui Pang ${ }^{1 *}$
}

\section{"Correspondence:}

phh2000@163.com

${ }^{1}$ College of Science, China

Agricultural University, Beijing,

P.R. China

\section{Springer}

\begin{abstract}
Using the monotone iterative technique, we investigate the existence of iterative positive solutions to a coupled system of fractional differential equations supplemented with multistrip and multipoint mixed boundary conditions. It is worth mentioning that the nonlinear terms of the system depend on the lower fractional-order derivatives of the unknown functions and the boundary conditions involve the combination of the multistrip fractional integral and the multipoint value of the unknown functions in $[0,1]$.
\end{abstract}

MSC: 26A33; 34B15; 34B18

Keywords: Fractional differential system; Multistrip and multipoint mixed boundary conditions; Green's function; Monotone iterative technique

\section{Introduction}

Fractional differential equations have attracted more and more scholars' attention since they are more widely used and realistic than integer-order differential equations. In the past few years the fractional boundary value problems are found to be popular in the research community because of their numerous applications in many disciplinary areas, such as optics, thermal, mechanics, control theory, nuclear physics, economics, signal and image processing, medicine, and so on [1-4]. To meet the practical application needs, many different theoretical approaches have been taken to study the existence, uniqueness, and multiplicity of solutions to fractional-order boundary value problems, for instance, the method of upper and lower solutions [5-9], the fixed point theory [10-13], the monotone iterative technique [14-19], the coincidence degree theory [20-22], etc. In comparison, the monotone iterative technique has more advantages, such as it not only proves the existence of positive solutions but also can obtain approximate solutions that can meet different accuracy requirements.

Meanwhile, recently, coupled fractional differential systems have also aroused great interest and developed rapidly. Many researchers established the existence of solutions by the class methods [22-25]. However, to the best of our knowledge, only few papers applied the monotone iterative technique to discuss the boundary value problem of coupled

(c) The Author(s) 2019. This article is distributed under the terms of the Creative Commons Attribution 4.0 International License (http://creativecommons.org/licenses/by/4.0/), which permits unrestricted use, distribution, and reproduction in any medium, provided you give appropriate credit to the original author(s) and the source, provide a link to the Creative Commons license, and indicate if changes were made. 
fractional differential systems [26-28]. To get more extensive results, different from the existing literature, we consider a generalized model that includes the nonlinear terms of the system depending on the lower fractional-order derivatives of the unknown functions and the boundary conditions involving a combination of the multistrip fractional integral and linear multipoint values of the unknown functions in $[0,1]$.

Based on these considerations, we investigate the existence of iterative positive solutions to the following fractional differential systems:

$$
\begin{cases}D_{t}^{\alpha_{1}} u(t)+f_{1}\left(t, u(t), v(t), D_{t}^{\gamma_{1}} u(t), D_{t}^{\gamma_{2}} v(t)\right)=0, & t \in(0,1), \\ D_{t}^{\alpha_{2}} v(t)+f_{2}\left(t, u(t), v(t), D_{t}^{\gamma_{1}} u(t), D_{t}^{\gamma_{2}} v(t)\right)=0, & t \in(0,1),\end{cases}
$$

with the coupled fractional-order integral and discrete mixed boundary conditions

$$
\left\{\begin{array}{l}
u(0)=u^{\prime}(0)=0, \quad u(1)=\sum_{i=1}^{m} \lambda_{1 i} I_{t}^{\beta_{1}} v\left(\xi_{i}\right)+\sum_{j=1}^{n} b_{j} v\left(\eta_{j}\right), \\
v(0)=v^{\prime}(0)=0, \quad v(1)=\sum_{i=1}^{m} \lambda_{2 i} i_{t}^{\beta_{2}} u\left(\xi_{i}\right)+\sum_{j=1}^{n} b_{j} u\left(\eta_{j}\right),
\end{array}\right.
$$

where $2<\alpha_{k} \leq 3,0<\gamma_{k}<\alpha_{k}-2,1<\beta_{k} \leq 2$ for $k=1,2 ; \lambda_{1 i}>0, \lambda_{2 i}>0,0<\xi_{i}<1$ for $i=$ $1,2, \ldots, m, b_{j} \geq 0,0<\eta_{j}<1$ for $j=1,2, \ldots, n$, and $D_{t}^{\alpha_{k}}$ and $D_{t}^{\gamma_{k}}$ are the standard RiemannLiouville fractional derivatives of orders $\alpha_{k}$ and $\gamma_{k}$ for $k=1,2$.

The coupled multistrip and multipoint mixed boundary conditions in (1.2) represent the value of unknown function $u(t)$ at the right end point $t=1$, which is equal to the sum of the values of the Riemann-Liouville fractional integral of the unknown function $v(t)$ on the subinterval $\left[0, \xi_{i}\right](i=1,2, \ldots, m)$ and the linear combination of discrete values of the unknown function $v(t)$ at $\eta_{j}(j=1,2, \ldots, n)$.

To apply the monotone iterative technique, we construct a concrete form of initial iterative function vector, which is a fractional power function vector satisfying the multiconstraints from the cone, the monotonicity of the complete continuous operator $T$, and the monotonicity of the lower fractional-order derivatives of $T$. The initial function vector of concise form could make the iteration process concise and effective.

\section{Preliminaries}

In this section, we present here the definitions, some lemmas from the theory of fractional calculus, and some auxiliary results for the proof of our main results.

Definition 2.1 ([1]) The Riemann-Liouville fractional integral of order $\alpha>0$ of a function $y:(0, \infty) \rightarrow \mathbb{R}$ is given by

$$
\left(I_{t}^{\alpha} y\right)(t)=\frac{1}{\Gamma(\alpha)} \int_{0}^{t}(t-s)^{\alpha-1} y(s) d s, \quad t>0,
$$

provided that the right-hand side is pointwise defined on $[0, \infty)$, where $\Gamma$ is the Euler gamma function defined as $\Gamma(\alpha)=\int_{0}^{\infty} t^{\alpha-1} e^{-t} d t$ for $\alpha>0$.

Definition 2.2 ([1]) The Riemann-Liouville fractional derivative of order $\alpha \geq 0$ for a function $y:(0, \infty) \rightarrow \mathbb{R}$ is given by

$$
\left(D_{t}^{\alpha} y\right)(t)=\frac{1}{\Gamma(n-\alpha)}\left(\frac{d}{d t}\right)^{n} \int_{0}^{t} y(s)(t-s)^{n-\alpha-1} d s, \quad t>0,
$$


where $n=[\alpha]+1$, provided that the right-hand side is pointwise defined on $[0, \infty)$. The notation $[\alpha]$ stands for the largest integer not greater than $\alpha$. We also denote the RiemannLiouville fractional derivative of $y$ by $D_{t}^{\alpha} y(t)$. If $\alpha=m \in \mathbb{N}$, then $D_{t}^{m} y(t)=y^{(m)}(t)$ for $t>0$, and if $\alpha=0$, then $D_{t}^{0} y(t)=y(t)$ for $t>0$.

Lemma 2.1 Let $\alpha>0$ and $n=[\alpha]+1$ for $\alpha \notin \mathbb{N}$, that is, $n$ is the smallest integer greater than or equal to $\alpha$. Then the solutions of the fractional differential equation $D_{t}^{\alpha} u(t)=0$, $0<t<1$, are

$$
u(t)=c_{1} t^{\alpha-1}+c_{2} t^{\alpha-2}+\cdots+c_{n} t^{\alpha-n}, \quad 0<t<1,
$$

where $c_{1}, c_{2}, \ldots, c_{n}$ are arbitrary real constants.

Lemma 2.2 Let $\alpha>0$, let $n$ be the smallest integer greater than or equal to $\alpha(n-1<\alpha \leq n)$, and let $y \in L^{1}(0,1)$. The solutions of the fractional equation $D_{t}^{\alpha} u(t)+y(t)=0,0<t<1$, are

$$
u(t)=-\frac{1}{\Gamma(\alpha)} \int_{0}^{t}(t-s)^{\alpha-1} y(s) d s+c_{1} t^{\alpha-1}+\cdots+c_{n} t^{\alpha-n}, \quad 0<t<1,
$$

where $c_{1}, c_{2}, \ldots, c_{n}$ are arbitrary real constants.

Remark 2.1 The following properties are useful for our discussion:

(i) As a basic example, we quote for $\alpha>-1$,

$$
D_{t}^{\gamma} t^{\alpha}=\frac{\Gamma(\alpha+1)}{\Gamma(\alpha-\gamma+1)} t^{\alpha-\gamma}
$$

(ii) $D_{t}^{\gamma} I_{t}^{\gamma} u(t)=u(t)$ for $u \in L^{1}(0,1), \gamma>0$;

(iii) Assume that $u \in L^{1}(0,1)$ with $\gamma>0$, then

$$
I_{t}^{\gamma}\left(D_{t}^{\gamma} u(t)\right)=u(t)+m_{1} t^{\gamma-1}+m_{2} t^{\gamma-2}+\cdots+m_{n} t^{\gamma-n}
$$

for some $m_{i} \in \mathbb{R}, i=1,2, \ldots, n$, where $n$ is the smallest integer greater than or equal to $\gamma$.

For convenience, we denote

$$
\left\{\begin{array}{l}
l_{1}=\sum_{i=1}^{m} \frac{\lambda_{2 i}}{\Gamma\left(\beta_{2}\right)} \int_{0}^{\xi_{i}}\left(\xi_{i}-s\right)^{\beta_{2}-1} s^{\alpha_{1}-1} d s+\sum_{j=1}^{n} b_{j} \eta_{j}^{\alpha_{1}-1}, \\
l_{2}=\sum_{i=1}^{m} \frac{\lambda_{1 i}}{\Gamma\left(\beta_{1}\right)} \int_{0}^{\xi_{i}}\left(\xi_{i}-s\right)^{\beta_{1}-1} s^{\alpha_{2}-1} d s+\sum_{j=1}^{n} b_{j} \eta_{j}^{\alpha_{2}-1} .
\end{array}\right.
$$

In the forthcoming analysis, we always need the following assumptions:

$\left(\mathrm{F}_{1}\right) 2<\alpha_{k}<3,1<\beta_{k} \leq 2$, and $0<\gamma_{k}<\alpha_{k}-2$ for $k=1,2$;

$\left(\mathrm{F}_{2}\right) 0<\eta_{j}, \xi_{i}<1, b_{j} \geq 0$, and $\lambda_{1 i}, \lambda_{2 i}>0$ for $i=1,2, \ldots, m, j=1,2, \ldots, n$;

(F $\left.\mathrm{F}_{3}\right) 1-l_{1} l_{2}>0$, where $l_{1}, l_{2}$ are defined by (2.1);

$\left(\mathrm{F}_{4}\right) f_{i}:[0,1] \times[0,+\infty)^{4} \rightarrow[0,+\infty), i=1,2$, are continuous functions.

Subject to BVP (1.1) and (1.2), we consider the corresponding linear boundary value problem as follows and establish expressions of the corresponding Green's functions. 
Lemma 2.3 Assume that $\left(\mathrm{F}_{1}\right)-\left(\mathrm{F}_{3}\right)$ hold. For $h_{1}, h_{2} \in L^{1}(0,1)$, the fractional differential system

$$
\begin{cases}D_{t}^{\alpha_{1}} u(t)+h_{1}(t)=0, & t \in(0,1) \\ D_{t}^{\alpha_{2}} v(t)+h_{2}(t)=0, & t \in(0,1)\end{cases}
$$

with boundary conditions (1.2) has the integral representation

$$
\left\{\begin{array}{l}
u(t)=\int_{0}^{1} K_{1}(t, s) h_{1}(s) d s+\int_{0}^{1} H_{1}(t, s) h_{2}(s) d s \\
v(t)=\int_{0}^{1} K_{2}(t, s) h_{2}(s) d s+\int_{0}^{1} H_{2}(t, s) h_{1}(s) d s
\end{array}\right.
$$

where

$$
\begin{aligned}
& K_{1}(t, s)=g_{1}(t, s)+\frac{l_{2} t^{\alpha_{1}-1}}{1-l_{1} l_{2}}\left[\sum_{i=1}^{m} \frac{\lambda_{2 i}}{\Gamma\left(\beta_{2}\right)} \int_{0}^{\xi_{i}}\left(\xi_{i}-\tau\right)^{\beta_{2}-1} g_{1}(\tau, s) d \tau+\sum_{j=1}^{n} b_{j} g_{1}\left(\eta_{j}, s\right)\right], \\
& H_{1}(t, s)=\frac{t^{\alpha_{1}-1}}{1-l_{1} l_{2}}\left[\sum_{i=1}^{m} \frac{\lambda_{1 i}}{\Gamma\left(\beta_{1}\right)} \int_{0}^{\xi_{i}}\left(\xi_{i}-\tau\right)^{\beta_{1}-1} g_{2}(\tau, s) d \tau+\sum_{j=1}^{n} b_{j} g_{2}\left(\eta_{j}, s\right)\right], \\
& K_{2}(t, s)=g_{2}(t, s)+\frac{l_{1} t^{\alpha_{2}-1}}{1-l_{1} l_{2}}\left[\sum_{i=1}^{m} \frac{\lambda_{1 i}}{\Gamma\left(\beta_{1}\right)} \int_{0}^{\xi_{i}}\left(\xi_{i}-\tau\right)^{\beta_{1}-1} g_{2}(\tau, s) d \tau+\sum_{j=1}^{n} b_{j} g_{2}\left(\eta_{j}, s\right)\right], \\
& H_{2}(t, s)=\frac{t^{\alpha_{2}-1}}{1-l_{1} l_{2}}\left[\sum_{i=1}^{m} \frac{\lambda_{2 i}}{\Gamma\left(\beta_{2}\right)} \int_{0}^{\xi_{i}}\left(\xi_{i}-\tau\right)^{\beta_{2}-1} g_{1}(\tau, s) d \tau+\sum_{j=1}^{n} b_{j} g_{1}\left(\eta_{j}, s\right)\right]
\end{aligned}
$$

and for $k=1,2$,

$$
g_{k}(t, s)=\frac{1}{\Gamma\left(\alpha_{k}\right)} \begin{cases}t^{\alpha_{k}-1}(1-s)^{\alpha_{k}-1}-(t-s)^{\alpha_{k}-1}, & 0 \leq s \leq t \leq 1, \\ t^{\alpha_{k}-1}(1-s)^{\alpha_{k}-1}, & 0 \leq t \leq s \leq 1 .\end{cases}
$$

Proof From Lemma 2.2 we can reduce (2.2) and (1.2) to the following equivalent integral equations:

$$
\left\{\begin{array}{l}
u(t)=-\int_{0}^{t} \frac{(t-s)^{\alpha_{1}-1}}{\Gamma\left(\alpha_{1}\right)} h_{1}(s) d s+c_{11} t^{\alpha_{1}-1}+c_{12} t^{\alpha_{1}-2}+c_{13} t^{\alpha_{1}-3} \\
v(t)=-\int_{0}^{t} \frac{(t-s)^{\alpha_{2}-1}}{\Gamma\left(\alpha_{2}\right)} h_{2}(s) d s+c_{21} t^{\alpha_{2}-1}+c_{22} t^{\alpha_{2}-2}+c_{23} t^{\alpha_{2}-3}
\end{array}\right.
$$

where $c_{12}, c_{13}, c_{22}, c_{23}$ are constants.

From $u(0)=u^{\prime}(0)=v(0)=v^{\prime}(0)=0$ we have $c_{12}=c_{13}=c_{22}=c_{23}=0$. Further, we use the right-hand side boundary conditions of (1.2) to reduce (2.7) to

$$
\left\{\begin{array}{l}
u(t)=t^{\alpha_{1}-1}\left[\sum_{i=1}^{m} \frac{\lambda_{1 i}}{\Gamma\left(\beta_{1}\right)} \int_{0}^{\xi_{i}}\left(\xi_{i}-s\right)^{\beta_{1}-1} v(s) d s+\sum_{j=1}^{n} b_{j} v\left(\eta_{j}\right)\right]+\int_{0}^{1} g_{1}(t, s) h_{1}(s) d s \\
v(t)=t^{\alpha_{2}-1}\left[\sum_{i=1}^{m} \frac{\lambda_{2 i}}{\Gamma\left(\beta_{2}\right)} \int_{0}^{\xi_{i}}\left(\xi_{i}-s\right)^{\beta_{2}-1} u(s) d s+\sum_{j=1}^{n} b_{j} u\left(\eta_{j}\right)\right]+\int_{0}^{1} g_{2}(t, s) h_{2}(s) d s .
\end{array}\right.
$$

Then we can get

$$
\sum_{i=1}^{m} \frac{\lambda_{1 i}}{\Gamma\left(\beta_{1}\right)} \int_{0}^{\xi_{i}}\left(\xi_{i}-s\right)^{\beta_{1}-1} v(s) d s+\sum_{j=1}^{n} b_{j} v\left(\eta_{j}\right)
$$


Zhao et al. Advances in Difference Equations

(2019) 2019:389

Page 5 of 23

$$
\begin{aligned}
& =\left[\sum_{i=1}^{m} \frac{\lambda_{2 i}}{\Gamma\left(\beta_{2}\right)} \int_{0}^{\xi_{i}}\left(\xi_{i}-s\right)^{\beta_{2}-1} u(s) d s+\sum_{j=1}^{n} b_{j} u\left(\eta_{j}\right)\right] \\
& \times\left[\sum_{i=1}^{m} \frac{\lambda_{1 i}}{\Gamma\left(\beta_{1}\right)} \int_{0}^{\xi_{i}}\left(\xi_{i}-s\right)^{\beta_{1}-1} s^{\alpha_{2}-1} d s+\sum_{j=1}^{n} b_{j} \eta_{j}^{\alpha_{2}-1}\right] \\
& +\sum_{j=1}^{n} b_{j} \int_{0}^{1} g_{2}\left(\eta_{j}, s\right) h_{2}(s) d s \\
& +\sum_{i=1}^{m} \frac{\lambda_{1 i}}{\Gamma\left(\beta_{1}\right)} \int_{0}^{\xi_{i}}\left(\xi_{i}-\tau\right)^{\beta_{1}-1} \int_{0}^{1} g_{2}(\tau, s) h_{2}(s) d s d \tau ; \\
& \sum_{i=1}^{m} \frac{\lambda_{2 i}}{\Gamma\left(\beta_{2}\right)} \int_{0}^{\xi_{i}}\left(\xi_{i}-s\right)^{\beta_{2}-1} u(s) d s+\sum_{j=1}^{n} b_{j} u\left(\eta_{j}\right) \\
& =\left[\sum_{i=1}^{m} \frac{\lambda_{1 i}}{\Gamma\left(\beta_{1}\right)} \int_{0}^{\xi_{i}}\left(\xi_{i}-s\right)^{\beta_{1}-1} v(s) d s+\sum_{j=1}^{n} b_{j} v\left(\eta_{j}\right)\right] \\
& \times\left[\sum_{i=1}^{m} \frac{\lambda_{2 i}}{\Gamma\left(\beta_{2}\right)} \int_{0}^{\xi_{i}}\left(\xi_{i}-s\right)^{\beta_{2}-1} s^{\alpha_{1}-1} d s+\sum_{j=1}^{n} b_{j} \eta_{j}^{\alpha_{1}-1}\right] \\
& +\sum_{j=1}^{n} b_{j} \int_{0}^{1} g_{1}\left(\eta_{j}, s\right) h_{1}(s) d s \\
& +\sum_{i=1}^{m} \frac{\lambda_{2 i}}{\Gamma\left(\beta_{2}\right)} \int_{0}^{\xi_{i}}\left(\xi_{i}-\tau\right)^{\beta_{2}-1} \int_{0}^{1} g_{1}(\tau, s) h_{1}(s) d s d \tau .
\end{aligned}
$$

Combining (2.1), (2.9), and (2.10), we can see that

$$
\begin{aligned}
\sum_{i=1}^{m} \frac{\lambda_{1 i}}{\Gamma\left(\beta_{1}\right)} \int_{0}^{\xi_{i}}\left(\xi_{i}-s\right)^{\beta_{1}-1} v(s) d s+\sum_{j=1}^{n} b_{j} v\left(\eta_{j}\right) \\
=\frac{1}{1-l_{1} l_{2}}\left[\left(\sum_{j=1}^{n} b_{j} \int_{0}^{1} g_{2}\left(\eta_{j}, s\right) h_{2}(s) d s\right.\right. \\
\left.+\sum_{i=1}^{m} \frac{\lambda_{1 i}}{\Gamma\left(\beta_{1}\right)} \int_{0}^{\xi_{i}}\left(\xi_{i}-\tau\right)^{\beta_{1}-1} \int_{0}^{1} g_{2}(\tau, s) h_{2}(s) d s d \tau\right) \\
+l_{2}\left(\sum_{j=1}^{n} b_{j} \int_{0}^{1} g_{1}\left(\eta_{j}, s\right) h_{1}(s) d s\right. \\
\left.\left.\quad+\sum_{i=1}^{m} \frac{\lambda_{2 i}}{\Gamma\left(\beta_{2}\right)} \int_{0}^{\xi_{i}}\left(\xi_{i}-\tau\right)^{\beta_{2}-1} \int_{0}^{1} g_{1}(\tau, s) h_{1}(s) d s d \tau\right)\right]
\end{aligned}
$$

where $l_{k}(k=1,2)$ are defined by (2.1). From (2.9) and (2.11) we have

$$
\begin{aligned}
u(t)= & \frac{t^{\alpha_{1}-1}}{1-l_{1} l_{2}}\left(\sum_{j=1}^{n} b_{j} \int_{0}^{1} g_{2}\left(\eta_{j}, s\right) h_{2}(s) d s\right. \\
& \left.+\sum_{i=1}^{m} \frac{\lambda_{1 i}}{\Gamma\left(\beta_{1}\right)} \int_{0}^{\xi_{i}}\left(\xi_{i}-\tau\right)^{\beta_{1}-1} \int_{0}^{1} g_{2}(\tau, s) h_{2}(s) d s d \tau\right)
\end{aligned}
$$


Zhao et al. Advances in Difference Equations

(2019) 2019:389

Page 6 of 23

$$
\begin{aligned}
& +\frac{l_{2} t^{\alpha_{1}-1}}{1-l_{1} l_{2}}\left(\sum_{j=1}^{n} b_{j} \int_{0}^{1} g_{1}\left(\eta_{j}, s\right) h_{1}(s) d s\right. \\
& \left.+\sum_{i=1}^{m} \frac{\lambda_{2 i}}{\Gamma\left(\beta_{2}\right)} \int_{0}^{\xi_{i}}\left(\xi_{i}-\tau\right)^{\beta_{2}-1} \int_{0}^{1} g_{1}(\tau, s) h_{1}(s) d s d \tau\right) \\
& +\int_{0}^{1} g_{1}(t, s) h_{1}(s) d s \\
& =\int_{0}^{1}\left[g_{1}(t, s)+\frac{l_{2} t^{\alpha_{1}-1}}{1-l_{1} l_{2}}\left(\sum_{j=1}^{n} b_{j} g_{1}\left(\eta_{j}, s\right)+\sum_{i=1}^{m} \frac{\lambda_{2 i}}{\Gamma\left(\beta_{2}\right)} \int_{0}^{\xi_{i}}\left(\xi_{i}-\tau\right)^{\beta_{2}-1} g_{1}(\tau, s) d \tau\right)\right] \\
& \quad \times h_{1}(s) d s \\
& +\int_{0}^{1} \frac{t^{\alpha_{1}-1}}{1-l_{1} l_{2}}\left(\sum_{j=1}^{n} b_{j} g_{2}\left(\eta_{j}, s\right)+\sum_{i=1}^{m} \frac{\lambda_{1 i}}{\Gamma\left(\beta_{1}\right)} \int_{0}^{\xi_{i}}\left(\xi_{i}-\tau\right)^{\beta_{1}-1} g_{2}(\tau, s) d \tau\right) h_{2}(s) d s \\
& =\int_{0}^{1} K_{1}(t, s) h_{1}(s) d s+\int_{0}^{1} H_{1}(t, s) h_{2}(s) d s .
\end{aligned}
$$

Similarly, we obtain

$$
v(t)=\int_{0}^{1} K_{2}(t, s) h_{2}(s) d s+\int_{0}^{1} H_{2}(t, s) h_{1}(s) d s,
$$

where $K_{2}(t, s)$ and $H_{2}(t, s)$ are given by $(2.5)$.

This completes the proof of the lemma.

Moreover, according to (2.3) and Remark 2.1, the fractional-order derivative of the solotion (2.3) can be expressed as

$$
\left\{\begin{array}{l}
D_{t}^{\gamma_{1}} u(t)=\int_{0}^{1} K_{3}(t, s) h_{1}(s) d s+\int_{0}^{1} H_{3}(t, s) h_{2}(s) d s \\
D_{t}^{\gamma_{2}} v(t)=\int_{0}^{1} K_{4}(t, s) h_{2}(s) d s+\int_{0}^{1} H_{4}(t, s) h_{1}(s) d s
\end{array}\right.
$$

where

$$
\begin{aligned}
K_{3}(t, s)= & D_{t}^{\gamma_{1}} g_{1}(t, s)+\frac{l_{2} t^{\alpha_{1}-\gamma_{1}-1} \Gamma\left(\alpha_{1}\right)}{\Gamma\left(\alpha_{1}-\gamma_{1}\right)\left(1-l_{1} l_{2}\right)}\left[\sum_{i=1}^{m} \frac{\lambda_{2 i}}{\Gamma\left(\beta_{2}\right)} \int_{0}^{\xi_{i}}\left(\xi_{i}-\tau\right)^{\beta_{2}-1} g_{1}(\tau, s) d \tau\right. \\
& \left.+\sum_{j=1}^{n} b_{j} g_{1}\left(\eta_{j}, s\right)\right], \\
H_{3}(t, s)= & \frac{\Gamma\left(\alpha_{1}\right) t^{\alpha_{1}-\gamma_{1}-1}}{\Gamma\left(\alpha_{1}-\gamma_{1}\right)\left(1-l_{1} l_{2}\right)}\left[\sum_{i=1}^{m} \frac{\lambda_{1 i}}{\Gamma\left(\beta_{1}\right)} \int_{0}^{\xi_{i}}\left(\xi_{i}-\tau\right)^{\beta_{1}-1} g_{2}(\tau, s) d \tau\right. \\
& \left.+\sum_{j=1}^{n} b_{j} g_{2}\left(\eta_{j}, s\right)\right], \\
K_{4}(t, s)= & D_{t}^{\gamma_{2}} g_{2}(t, s)+\frac{l_{1} t^{\alpha_{2}-\gamma_{2}-1} \Gamma\left(\alpha_{2}\right)}{\Gamma\left(\alpha_{2}-\gamma_{2}\right)\left(1-l_{1} l_{2}\right)}\left[\sum_{i=1}^{m} \frac{\lambda_{1 i}}{\Gamma\left(\beta_{1}\right)} \int_{0}^{\xi_{i}}\left(\xi_{i}-\tau\right)^{\beta_{1}-1} g_{2}(\tau, s) d \tau\right.
\end{aligned}
$$




$$
\begin{gathered}
\left.+\sum_{j=1}^{n} b_{j} g_{2}\left(\eta_{j}, s\right)\right] \\
H_{4}(t, s)=\frac{\Gamma\left(\alpha_{2}\right) t^{\alpha_{2}-\gamma_{2}-1}}{\Gamma\left(\alpha_{2}-\gamma_{2}\right)\left(1-l_{1} l_{2}\right)}\left[\sum_{i=1}^{m} \frac{\lambda_{2 i}}{\Gamma\left(\beta_{2}\right)} \int_{0}^{\xi_{i}}\left(\xi_{i}-\tau\right)^{\beta_{2}-1} g_{1}(\tau, s) d \tau+\sum_{j=1}^{n} b_{j} g_{1}\left(\eta_{j}, s\right)\right],
\end{gathered}
$$

and for $k, j=1,2$,

$$
D_{t}^{\gamma_{j}} g_{k}(t, s)=\frac{1}{\Gamma\left(\alpha_{k}-\gamma_{j}\right)} \begin{cases}t^{\alpha_{k}-\gamma_{j}-1}(1-s)^{\alpha_{k}-1}-(t-s)^{\alpha_{k}-\gamma_{j}-1}, & 0 \leq s \leq t \leq 1, \\ t^{\alpha_{k}-\gamma_{j}-1}(1-s)^{\alpha_{k}-1}, & 0 \leq t \leq s \leq 1 .\end{cases}
$$

Lemma 2.4 Assume that $\left(\mathrm{F}_{1}\right)$ holds. Then the functions $g_{k}(t, s)$ and $D_{t}^{r_{j}} g_{k}(t, s), k, j=1,2$, defined by (2.6) and (2.14) have the following properties:

(1) $0 \leq g_{k}(t, s) \leq \frac{1}{\Gamma\left(\alpha_{k}\right)} t^{\alpha_{k}-1}\left[(1-s)^{\alpha_{k}-1}+1\right]$ for $t, s \in[0,1]$;

(2) $\left|D_{t}^{r_{j}} g_{k}(t, s)\right| \leq \frac{1}{\Gamma\left(\alpha_{k}-\gamma_{j}\right)} t^{\alpha_{k}-\gamma_{j}-1}\left[(1-s)^{\alpha_{k}-1}+1\right]$ for $t, s \in[0,1]$.

Proof (1) For $0 \leq s \leq t \leq 1$, we have

$$
\begin{aligned}
g_{k}(t, s) & =\frac{1}{\Gamma\left(\alpha_{k}\right)}\left(t^{\alpha_{k}-1}(1-s)^{\alpha_{k}-1}-(t-s)^{\alpha_{k}-1}\right) \\
& =\frac{1}{\Gamma\left(\alpha_{k}\right)}\left((t-t s)^{\alpha_{k}-1}-(t-s)^{\alpha_{k}-1}\right) \\
& \geq 0 \\
g_{k}(t, s) & =\frac{1}{\Gamma\left(\alpha_{k}\right)}\left(t^{\alpha_{k}-1}(1-s)^{\alpha_{k}-1}-(t-s)^{\alpha_{k}-1}\right) \\
& \leq \frac{1}{\Gamma\left(\alpha_{k}\right)} t^{\alpha_{k}-1}(1-s)^{\alpha_{k}-1} \\
& \leq \frac{1}{\Gamma\left(\alpha_{k}\right)} t^{\alpha_{k}-1}\left[(1-s)^{\alpha_{k}-1}+1\right] .
\end{aligned}
$$

For $0 \leq t \leq s \leq 1$, we have

$$
0 \leq g_{k}(t, s)=\frac{1}{\Gamma\left(\alpha_{k}\right)} t^{\alpha_{k}-1}(1-s)^{\alpha_{k}-1} \leq \frac{1}{\Gamma\left(\alpha_{k}\right)} t^{\alpha_{k}-1}\left[(1-s)^{\alpha_{k}-1}+1\right] .
$$

(2) For $0 \leq s \leq t \leq 1$, we get

$$
\begin{aligned}
\left|D_{t}^{r_{j}} g_{k}(t, s)\right| & =\left|\frac{1}{\Gamma\left(\alpha_{k}-\gamma_{j}\right)}\left[t^{\alpha_{k}-\gamma_{j}-1}(1-s)^{\alpha_{k}-1}-(t-s)^{\alpha_{k}-\gamma_{j}-1}\right]\right| \\
& \leq \frac{1}{\Gamma\left(\alpha_{k}-\gamma_{j}\right)}\left[t^{\alpha_{k}-\gamma_{j}-1}(1-s)^{\alpha_{k}-1}+(t-s)^{\alpha_{k}-\gamma_{j}-1}\right] \\
& \leq \frac{1}{\Gamma\left(\alpha_{k}-\gamma_{j}\right)}\left[t^{\alpha_{k}-\gamma_{j}-1}(1-s)^{\alpha_{k}-1}+t^{\alpha_{k}-\gamma_{j}-1}\right] \\
& =\frac{1}{\Gamma\left(\alpha_{k}-\gamma_{j}\right)} t^{\alpha_{k}-\gamma_{j}-1}\left[(1-s)^{\alpha_{k}-1}+1\right] .
\end{aligned}
$$


For $0 \leq t \leq s \leq 1$, we get

$$
\begin{aligned}
\left|D_{t}^{\gamma_{j}} g_{k}(t, s)\right| & =\frac{1}{\Gamma\left(\alpha_{k}-\gamma_{j}\right)} t^{\alpha_{k}-\gamma_{j}-1}(1-s)^{\alpha_{k}-1} \\
& \leq \frac{1}{\Gamma\left(\alpha_{k}-\gamma_{j}\right)} t^{\alpha_{k}-\gamma_{j}-1}\left[(1-s)^{\alpha_{k}-1}+1\right] .
\end{aligned}
$$

This completes the proof of the lemma.

For convenience, we denote

$$
\begin{aligned}
& \varrho_{1}=\frac{1}{\Gamma\left(\alpha_{1}\right)}\left[1+\frac{l_{2}}{1-l_{1} l_{2}}\left(\sum_{i=1}^{m} \frac{\lambda_{2 i}}{\Gamma\left(\beta_{2}\right)} \int_{0}^{\xi_{i}}\left(\xi_{i}-\tau\right)^{\beta_{2}-1} \tau^{\alpha_{1}-1} d \tau+\sum_{j=1}^{n} b_{j} \eta_{j}^{\alpha_{1}-1}\right)\right], \\
& \varrho_{2}=\frac{1}{\Gamma\left(\alpha_{2}\right)}\left[1+\frac{l_{1}}{1-l_{1} l_{2}}\left(\sum_{i=1}^{m} \frac{\lambda_{1 i}}{\Gamma\left(\beta_{1}\right)} \int_{0}^{\xi_{i}}\left(\xi_{i}-\tau\right)^{\beta_{1}-1} \tau^{\alpha_{2}-1} d \tau+\sum_{j=1}^{n} b_{j} \eta_{j}^{\alpha_{2}-1}\right)\right], \\
& \rho_{1}=\frac{1}{\Gamma\left(\alpha_{2}\right)\left(1-l_{1} l_{2}\right)}\left[\sum_{i=1}^{m} \frac{\lambda_{1 i}}{\Gamma\left(\beta_{1}\right)} \int_{0}^{\xi_{i}}\left(\xi_{i}-\tau\right)^{\beta_{1}-1} \tau^{\alpha_{2}-1} d \tau+\sum_{j=1}^{n} b_{j} \eta_{j}^{\alpha_{2}-1}\right], \\
& \rho_{2}=\frac{1}{\Gamma\left(\alpha_{1}\right)\left(1-l_{1} l_{2}\right)}\left[\sum_{i=1}^{m} \frac{\lambda_{2 i}}{\Gamma\left(\beta_{2}\right)} \int_{0}^{\xi_{i}}\left(\xi_{i}-\tau\right)^{\beta_{2}-1} \tau^{\alpha_{1}-1} d \tau+\sum_{j=1}^{n} b_{j} \eta_{j}^{\alpha_{1}-1}\right] .
\end{aligned}
$$

Lemma 2.5 Assume that $\left(\mathrm{F}_{1}\right)-\left(\mathrm{F}_{3}\right)$ hold. Then for $(t, s) \in[0,1] \times[0,1]$, the functions $K_{i}(t, s)$ and $H_{i}(t, s), i=1,2,3,4$, defined by (2.4), (2.5), and (2.13) satisfy the following inequalities:

(1) $0 \leq K_{1}(t, s) \leq t^{\alpha_{1}-1}\left[(1-s)^{\alpha_{1}-1}+1\right] \varrho_{1}$,

$$
0 \leq K_{2}(t, s) \leq t^{\alpha_{2}-1}\left[(1-s)^{\alpha_{2}-1}+1\right] \varrho_{2}
$$$$
\left|K_{3}(t, s)\right| \leq \frac{\Gamma\left(\alpha_{1}\right)}{\Gamma\left(\alpha_{1}-\gamma_{1}\right)} t^{\alpha_{1}-\gamma_{1}-1}\left[(1-s)^{\alpha_{1}-1}+1\right] \varrho_{1},
$$$$
\left|K_{4}(t, s)\right| \leq \frac{\Gamma\left(\alpha_{2}\right)}{\Gamma\left(\alpha_{2}-\gamma_{2}\right)} t^{\alpha_{2}-\gamma_{2}-1}\left[(1-s)^{\alpha_{2}-1}+1\right] \varrho_{2} ;
$$

(2) $0 \leq H_{1}(t, s) \leq t^{\alpha_{1}-1}\left[(1-s)^{\alpha_{2}-1}+1\right] \rho_{1}$,

$$
\begin{aligned}
& 0 \leq H_{2}(t, s) \leq t^{\alpha_{2}-1}\left[(1-s)^{\alpha_{1}-1}+1\right] \rho_{2}, \\
& 0 \leq H_{3}(t, s) \leq \frac{\Gamma\left(\alpha_{1}\right)}{\Gamma\left(\alpha_{1}-\gamma_{1}\right)} t^{\alpha_{1}-\gamma_{1}-1}\left[(1-s)^{\alpha_{2}-1}+1\right] \rho_{1}, \\
& 0 \leq H_{4}(t, s) \leq \frac{\Gamma\left(\alpha_{2}\right)}{\Gamma\left(\alpha_{2}-\gamma_{2}\right)} t^{\alpha_{2}-\gamma_{2}-1}\left[(1-s)^{\alpha_{1}-1}+1\right] \rho_{2} .
\end{aligned}
$$

Proof (1) According to $\left(\mathrm{F}_{3}\right)$, Lemma 2.4, and the definition of $K_{i}(t, s)$, we obtain

$$
\begin{aligned}
0 & \leq K_{1}(t, s)=g_{1}(t, s)+\frac{l_{2} t^{\alpha_{1}-1}}{1-l_{1} l_{2}}\left[\sum_{i=1}^{m} \frac{\lambda_{2 i}}{\Gamma\left(\beta_{2}\right)} \int_{0}^{\xi_{i}}\left(\xi_{i}-\tau\right)^{\beta_{2}-1} g_{1}(\tau, s) d \tau+\sum_{j=1}^{n} b_{j} g_{1}\left(\eta_{j}, s\right)\right] \\
& \leq \frac{1}{\Gamma\left(\alpha_{1}\right)} t^{\alpha_{1}-1}\left[(1-s)^{\alpha_{1}-1}+1\right]
\end{aligned}
$$


Zhao et al. Advances in Difference Equations

(2019) 2019:389

Page 9 of 23

$$
\begin{aligned}
& +\frac{l_{2} t^{\alpha_{1}-1}}{1-l_{1} l_{2}}\left[\sum_{i=1}^{m} \frac{\lambda_{2 i}}{\Gamma\left(\beta_{2}\right) \Gamma\left(\alpha_{1}\right)} \int_{0}^{\xi_{i}}\left(\xi_{i}-\tau\right)^{\beta_{2}-1} \tau^{\alpha_{1}-1}\left[(1-s)^{\alpha_{1}-1}+1\right] d \tau\right. \\
& \left.+\frac{1}{\Gamma\left(\alpha_{1}\right)} \sum_{j=1}^{n} b_{j} \eta_{j}^{\alpha_{1}-1}\left[(1-s)^{\alpha_{1}-1}+1\right]\right] \\
& =t^{\alpha_{1}-1}\left[(1-s)^{\alpha_{1}-1}+1\right] \frac{1}{\Gamma\left(\alpha_{1}\right)}\left[1+\frac{l_{2}}{1-l_{1} l_{2}}\left(\sum_{i=1}^{m} \frac{\lambda_{2 i}}{\Gamma\left(\beta_{2}\right)} \int_{0}^{\xi_{i}}\left(\xi_{i}-\tau\right)^{\beta_{2}-1} \tau^{\alpha_{1}-1} d \tau\right.\right. \\
& \left.\left.\quad+\sum_{j=1}^{n} b_{j} \eta_{j}^{\alpha_{1}-1}\right)\right] \\
& =t^{\alpha_{1}-1}\left[(1-s)^{\alpha_{1}-1}+1\right] \varrho_{1}
\end{aligned}
$$

and

$$
\begin{aligned}
\left|K_{3}(t, s)\right|= & \mid D_{t}^{\gamma_{1}} g_{1}(t, s)+\frac{l_{2} t^{\alpha_{1}-\gamma_{1}-1} \Gamma\left(\alpha_{1}\right)}{\Gamma\left(\alpha_{1}-\gamma_{1}\right)\left(1-l_{1} l_{2}\right)}\left[\sum_{i=1}^{m} \frac{\lambda_{2 i}}{\Gamma\left(\beta_{2}\right)} \int_{0}^{\xi_{i}}\left(\xi_{i}-\tau\right)^{\beta_{2}-1} g_{1}(\tau, s) d \tau\right. \\
& \left.+\sum_{j=1}^{n} b_{j} g_{1}\left(\eta_{j}, s\right)\right] \mid \\
\leq & \frac{1}{\Gamma\left(\alpha_{1}-\gamma_{1}\right)} t^{\alpha_{1}-\gamma_{1}-1}\left[(1-s)^{\alpha_{1}-1}+1\right] \\
& +\frac{l_{2} t^{\alpha_{1}-\gamma_{1}-1} \Gamma\left(\alpha_{1}\right)}{\Gamma\left(\alpha_{1}-\gamma_{1}\right)\left(1-l_{1} l_{2}\right)}\left[\sum_{i=1}^{m} \frac{\lambda_{2 i}}{\Gamma\left(\beta_{2}\right) \Gamma\left(\alpha_{1}\right)} \int_{0}^{\xi_{i}}\left(\xi_{i}-\tau\right)^{\beta_{2}-1}\right. \\
& \left.\times \tau^{\alpha_{1}-1}\left[(1-s)^{\alpha_{1}-1}+1\right] d \tau+\frac{1}{\Gamma\left(\alpha_{1}\right)} \sum_{j=1}^{n} b_{j} \eta_{j}^{\alpha_{1}-1}\left[(1-s)^{\alpha_{1}-1}+1\right]\right] \\
\leq & \frac{\Gamma\left(\alpha_{1}\right)}{\Gamma\left(\alpha_{1}-\gamma_{1}\right)} t^{\alpha_{1}-\gamma_{1}-1}\left[(1-s)^{\alpha_{1}-1}+\right] \varrho_{1},
\end{aligned}
$$

where $\varrho_{1}$ is defined by $(2.15)$.

Similarly, we get

$$
\begin{aligned}
& 0 \leq K_{2}(t, s) \leq t^{\alpha_{2}-1}\left[(1-s)^{\alpha_{2}-1}+1\right] \varrho_{2}, \\
& \left|K_{4}(t, s)\right| \leq \frac{\Gamma\left(\alpha_{2}\right)}{\Gamma\left(\alpha_{2}-\gamma_{2}\right)} t^{\alpha_{2}-\gamma_{2}-1}\left[(1-s)^{\alpha_{2}-1}+1\right] \varrho_{2},
\end{aligned}
$$

where $\varrho_{2}$ is defined by (2.16).

(2) According to $\left(\mathrm{F}_{3}\right)$, Lemma 2.4, and the definition of $H_{k}(t, s)(k=1,2,3,4)$, we infer that

$$
\begin{aligned}
0 & \leq H_{1}(t, s)=\frac{t^{\alpha_{1}-1}}{1-l_{1} l_{2}}\left[\sum_{i=1}^{m} \frac{\lambda_{1 i}}{\Gamma\left(\beta_{1}\right)} \int_{0}^{\xi_{i}}\left(\xi_{i}-\tau\right)^{\beta_{1}-1} g_{2}(\tau, s) d \tau+\sum_{j=1}^{n} b_{j} g_{2}\left(\eta_{j}, s\right)\right] \\
& \leq \frac{t^{\alpha_{1}-1}}{1-l_{1} l_{2}}\left[\sum_{i=1}^{m} \frac{\lambda_{1 i}}{\Gamma\left(\beta_{1}\right) \Gamma\left(\alpha_{2}\right)} \int_{0}^{\xi_{i}}\left(\xi_{i}-\tau\right)^{\beta_{1}-1} \tau^{\alpha_{2}-1}\left[(1-s)^{\alpha_{2}-1}+1\right] d \tau\right.
\end{aligned}
$$




$$
\begin{aligned}
& \left.+\frac{1}{\Gamma\left(\alpha_{2}\right)} \sum_{j=1}^{n} b_{j} \eta_{j}^{\alpha_{2}-1}\left[(1-s)^{\alpha_{2}-1}+1\right]\right] \\
= & t^{\alpha_{1}-1}\left[(1-s)^{\alpha_{2}-1}+1\right] \frac{1}{\Gamma\left(\alpha_{2}\right)\left(1-l_{1} l_{2}\right)}\left[\sum_{i=1}^{m} \frac{\lambda_{1 i}}{\Gamma\left(\beta_{1}\right)} \int_{0}^{\xi_{i}}\left(\xi_{i}-\tau\right)^{\beta_{1}-1} \tau^{\alpha_{2}-1} d \tau\right. \\
& \left.+\sum_{j=1}^{n} b_{j} \eta_{j}^{\alpha_{2}-1}\right] \\
= & t^{\alpha_{1}-1}\left[(1-s)^{\alpha_{2}-1}+1\right] \rho_{1}
\end{aligned}
$$

and

$$
\begin{aligned}
0 \leq & H_{3}(t, s) \\
= & \frac{\Gamma\left(\alpha_{1}\right) t^{\alpha_{1}-\gamma_{1}-1}}{\Gamma\left(\alpha_{1}-\gamma_{1}\right)\left(1-l_{1} l_{2}\right)}\left[\sum_{i=1}^{m} \frac{\lambda_{1 i}}{\Gamma\left(\beta_{1}\right)} \int_{0}^{\xi_{i}}\left(\xi_{i}-\tau\right)^{\beta_{1}-1} g_{2}(\tau, s) d \tau+\sum_{j=1}^{n} b_{j} g_{2}\left(\eta_{j}, s\right)\right] \\
\leq & \frac{\Gamma\left(\alpha_{1}\right) t^{\alpha_{1}-\gamma_{1}-1}}{\Gamma\left(\alpha_{1}-\gamma_{1}\right)\left(1-l_{1} l_{2}\right)}\left[\sum_{i=1}^{m} \frac{\lambda_{1 i}}{\Gamma\left(\beta_{1}\right) \Gamma\left(\alpha_{2}\right)} \int_{0}^{\xi_{i}}\left(\xi_{i}-\tau\right)^{\beta_{1}-1} \tau^{\alpha_{2}-1}\left[(1-s)^{\alpha_{2}-1}+1\right] d \tau\right. \\
& \left.+\frac{1}{\Gamma\left(\alpha_{2}\right)} \sum_{j=1}^{n} b_{j} \eta_{j}^{\alpha_{2}-1}\left[(1-s)^{\alpha_{2}-1}+1\right]\right] \\
= & \frac{\Gamma\left(\alpha_{1}\right)}{\Gamma\left(\alpha_{1}-\gamma_{1}\right)} t^{\alpha_{1}-\gamma_{1}-1}\left[(1-s)^{\alpha_{2}-1}+1\right] \rho_{1},
\end{aligned}
$$

where $\rho_{1}$ is defined by (2.17). Analogously, we get

$$
\begin{aligned}
& 0 \leq H_{2}(t, s) \leq t^{\alpha_{2}-1}\left[(1-s)^{\alpha_{1}-1}+1\right] \rho_{2}, \\
& 0 \leq H_{4}(t, s) \leq \frac{\Gamma\left(\alpha_{2}\right)}{\Gamma\left(\alpha_{2}-\gamma_{2}\right)} t^{\alpha_{2}-\gamma_{2}-1}\left[(1-s)^{\alpha_{1}-1}+1\right] \rho_{2},
\end{aligned}
$$

where $\rho_{2}$ is defined by (2.18).

This completes the proof of the lemma.

Let $X=\left\{u \mid u \in C[0,1]\right.$ and $\left.D_{t}^{\gamma_{1}} u \in C[0,1]\right\}$ be endowed with the norm

$$
\|u\|=\max \left\{\|u\|_{0},\left\|D_{t}^{\gamma_{1}} u\right\|_{0}\right\}
$$

where $\|u\|_{0}=\max _{t \in[0,1]}|u(t)|$ and $\left\|D_{t}^{\gamma_{1}} u\right\|_{0}=\max _{t \in[0,1]}\left|D_{t}^{\gamma_{1}} u(t)\right|$. Also let $Y=\{v \mid v \in$ $C[0,1]$ and $\left.D_{t}^{\gamma_{2}} v \in C[0,1]\right\}$ be endowed with the norm

$$
\|v\|=\max \left\{\|v\|_{0},\left\|D_{t}^{\gamma_{2}} v\right\|_{0}\right\}
$$

where $\|v\|_{0}=\max _{t \in[0,1]}|v(t)|$ and $\left\|D_{t}^{\gamma_{1}} v\right\|_{0}=\max _{t \in[0,1]}\left|D_{t}^{\gamma_{1}} v(t)\right|$. We introduce the product space $(X \times Y,\|(u, v)\|)$ endowed with the norm $\|(u, v)\|=\max \{\|u\|,\|v\|\}$ and define a partial order over the product space:

$$
\left(\begin{array}{l}
u_{1} \\
v_{1}
\end{array}\right) \geq\left(\begin{array}{l}
u_{2} \\
v_{2}
\end{array}\right)
$$

if $u_{1}(t) \geq u_{2}(t), v_{1}(t) \geq v_{2}(t), D_{t}^{\gamma_{1}} u_{1}(t) \geq D_{t}^{\gamma_{1}} u_{2}(t)$, and $D_{t}^{\gamma_{2}} v_{1}(t) \geq D_{t}^{\gamma_{2}} v_{2}(t), t \in[0,1]$. 
Lemma $2.6(X \times Y,\|(u, v)\|)$ is a Banach space.

Proof Let $\left\{u_{n}\right\}_{n=1}^{\infty}$ be a Cauchy sequence in the space $(X,\|\cdot\|)$. It is obvious that $\left\{u_{n}\right\}_{n=1}^{\infty}$ and $\left\{D_{t}^{\gamma_{1}} u_{n}\right\}_{n=1}^{\infty}$ are Cauchy sequences in the space $C[0,1]$. Let us assume that $\left\{u_{n}\right\}_{n=1}^{\infty}$ and $\left\{D_{t}^{\gamma_{1}} u_{n}\right\}_{n=1}^{\infty}$ uniformly converge on $[0,1]$ to $u \in C[0,1]$ and $w \in C[0,1]$. Now we should prove that $w=D_{t}^{\gamma_{1}} u$.

For $t \in[0,1]$, we have

$$
\begin{aligned}
\left|I_{t}^{\gamma_{1}} D_{t}^{\gamma_{1}} u_{n}(t)-I_{t}^{\gamma_{1}} w(t)\right| & \leq \frac{1}{\Gamma\left(\gamma_{1}\right)} \int_{0}^{t}(t-s)^{\gamma_{1}-1}\left|D_{t}^{\gamma_{1}} u_{n}(s)-w(s)\right| d s \\
& \leq \frac{1}{\Gamma\left(\gamma_{1}+1\right)} \max _{s \in[0,1]}\left|D_{t}^{\gamma_{1}} u_{n}(s)-w(s)\right| .
\end{aligned}
$$

In view of the convergence of $\left\{u_{n}\right\}_{n=1}^{\infty}$, we obtain that $\lim _{n \rightarrow \infty} I_{t}^{\gamma_{1}} D_{t}^{\gamma_{1}} u_{n}(t)=I_{t}^{\gamma_{1}} w(t)$ uniformly for $t \in[0,1]$. Otherwise, by Remark 2.1 we have $I_{t}^{\gamma_{1}} D_{t}^{\gamma_{1}} u_{n}(t)=u_{n}(t)$ for $t \in[0,1]$. These two facts imply that

$$
\lim _{n \rightarrow \infty} I_{t}^{\gamma_{1}} D_{t}^{\gamma_{1}} u_{n}(t)=\lim _{n \rightarrow \infty} u_{n}(t)=I_{t}^{\gamma_{1}} w(t) \quad \text { for } t \in[0,1]
$$

Combining this with $\lim _{n \rightarrow \infty} u_{n}(t)=u(t)$ for $t \in[0,1]$, we have

$$
u(t)=I_{t}^{\gamma_{1}} w(t) \quad \text { for } t \in[0,1]
$$

Taking the $\gamma_{1}$ th-order derivatives of both sides of equation (2.19), in consequence, we have

$$
D_{t}^{\gamma_{1}} I_{t}^{\gamma_{1}} w(t)=D_{t}^{\gamma_{1}} u(t) \quad \text { for } t \in[0,1]
$$

By Remark 2.1 this leads to

$$
w(t)=D_{t}^{\gamma_{1}} u(t) \quad \text { for } t \in[0,1]
$$

which proves that $(X,\|\cdot\|)$ is a Banach space.

In the same way, we can prove that $(Y,\|\cdot\|)$ is a Banach space. Moreover, the product space $(X \times Y,\|(u, v)\|)$ is also a Banach space with the norm $\|(u, v)\|=\max \{\|u\|,\|v\|\}$.

The proof of the lemma is completed.

Further, we define the cone $P \subset X \times Y$ by $P=\left\{(u, v) \in X \times Y: u(t) \geq 0, v(t) \geq 0, D_{t}^{\gamma_{1}} u(t) \geq\right.$ $\left.0, D_{t}^{\gamma_{2}} v(t) \geq 0, t \in[0,1]\right\}$. For all $(u, v) \in P$, in view of Lemma 2.3 and $\left(\mathrm{F}_{4}\right)$, let $T: P \rightarrow P$ be the operator defined by

$$
T(u, v)(t)=\left(\begin{array}{l}
T_{1}(u, v)(t) \\
T_{2}(u, v)(t)
\end{array}\right)
$$

where

$$
T_{1}(u, v)(t)=\int_{0}^{1} K_{1}(t, s) f_{1(u, v)}(s) d s+\int_{0}^{1} H_{1}(t, s) f_{2(u, v)}(s) d s,
$$




$$
T_{2}(u, v)(t)=\int_{0}^{1} K_{2}(t, s) f_{2(u, v)}(s) d s+\int_{0}^{1} H_{2}(t, s) f_{1(u, v)}(s) d s
$$

and for convenience, we set

$$
\left\{\begin{array}{l}
f_{1(u, v)}(s) \triangleq f_{1}\left(s, u(s), v(s), D_{t}^{\gamma_{1}} u(s), D_{t}^{\gamma_{2}} v(s)\right) \\
f_{2(u, v)}(s) \triangleq f_{2}\left(s, u(s), v(s), D_{t}^{\gamma_{1}} u(s), D_{t}^{\gamma_{2}} v(s)\right)
\end{array}\right.
$$

Also, from (2.12) we have

$$
\begin{aligned}
& D_{t}^{\gamma_{1}} T_{1}(u, v)(t)=\int_{0}^{1} K_{3}(t, s) f_{1(u, v)}(s) d s+\int_{0}^{1} H_{3}(t, s) f_{2(u, v)}(s) d s, \\
& D_{t}^{\gamma_{2}} T_{2}(u, v)(t)=\int_{0}^{1} K_{4}(t, s) f_{2(u, v)}(s) d s+\int_{0}^{1} H_{4}(t, s) f_{1(u, v)}(s) d s .
\end{aligned}
$$

Lemma 2.7 The operator $T: P \rightarrow P$ is completely continuous.

Proof By the continuity of the functions $K_{1}(t, s)-K_{4}(t, s), H_{1}(t, s)-H_{4}(t, s)$, and $f_{1}$ and $f_{2}$ the operator $T$ is continuous.

Then we show $T$ is uniformly bounded. Let $\Omega \subset P$ be bounded. There exists a positive constant $M$ satisfying the inequality

$$
\max \left\{\left|f_{1(u, v)}(t)\right|,\left|f_{2(u, v)}(t)\right|\right\} \leq M, \quad \forall(u, v) \in \Omega
$$

For any $(u, v) \in \Omega$, combining Lemma 2.5 with (2.20), (2.22), and (2.24), we get

$$
\begin{aligned}
\left|T_{1}(u, v)(t)\right| & \leq\left|M \int_{0}^{1} K_{1}(t, s) d s+M \int_{0}^{1} H_{1}(t, s) d s\right| \\
& \leq M \int_{0}^{1}\left\{t^{\alpha_{1}-1}\left[(1-s)^{\alpha_{1}-1}+1\right] \varrho_{1}+t^{\alpha_{1}-1}\left[(1-s)^{\alpha_{2}-1}+1\right] \rho_{1}\right\} d s \\
& \leq M \int_{0}^{1}\left\{\left[(1-s)^{\alpha_{1}-1}+1\right] \varrho_{1}+\left[(1-s)^{\alpha_{2}-1}+1\right] \rho_{1}\right\} d s
\end{aligned}
$$

and

$$
\begin{aligned}
& \left|D_{t}^{\gamma_{1}} T_{1}(u, v)(t)\right| \\
& \quad \leq\left|M \int_{0}^{1} K_{3}(t, s) d s+M \int_{0}^{1} H_{3}(t, s) d s\right| \\
& \quad \leq \frac{M \Gamma\left(\alpha_{1}\right)}{\Gamma\left(\alpha_{1}-\gamma_{1}\right)} \int_{0}^{1}\left\{t^{\alpha_{1}-\gamma_{1}-1}\left[(1-s)^{\alpha_{1}-1}+1\right] \varrho_{1}+t^{\alpha_{1}-\gamma_{1}-1}\left[(1-s)^{\alpha_{2}-1}+1\right] \rho_{1}\right\} d s \\
& \quad \leq \frac{M \Gamma\left(\alpha_{1}\right)}{\Gamma\left(\alpha_{1}-\gamma_{1}\right)} \int_{0}^{1}\left\{\left[(1-s)^{\alpha_{1}-1}+1\right] \varrho_{1}+\left[(1-s)^{\alpha_{2}-1}+1\right] \rho_{1}\right\} d s,
\end{aligned}
$$

which implies that $\left\|T_{1}(u, v)\right\|$ is uniformly bounded. Further, we get that $\left\|T_{2}(u, v)\right\|$ is also uniformly bounded. Thus it follows from the above inequalities that the operator $T$ is uniformly bounded. 
Next, we show that $T$ is equicontinuous. For any $(u, v) \in \Omega$ and $t_{1}, t_{2} \in[0,1]$, in view of Lemma 2.5, (2.20), (2.22), and (2.24), we infer that

$$
\begin{aligned}
& \left|T_{1}(u, v)\left(t_{2}\right)-T_{1}(u, v)\left(t_{1}\right)\right| \\
& \leq\left|M \int_{0}^{1}\left(K_{1}\left(t_{2}, s\right)-K_{1}\left(t_{1}, s\right)\right) d s\right|+\left|M \int_{0}^{1}\left(H_{1}\left(t_{2}, s\right)-H_{1}\left(t_{1}, s\right)\right) d s\right| \\
& \leq M \int_{0}^{1}\left\{\left|\left(t_{2}^{\alpha_{1}-1}-t_{1}^{\alpha_{1}-1}\right)\left[(1-s)^{\alpha_{1}-1}+1\right]\right| \varrho_{1}\right. \\
& \left.\quad+\left|\left(t_{2}^{\alpha_{1}-1}-t_{1}^{\alpha_{1}-1}\right)\left[(1-s)^{\alpha_{2}-1}+1\right]\right| \rho_{1}\right\} d s .
\end{aligned}
$$

Applying the mean value theorem, we have the inequality

$$
t_{2}^{\alpha-1}-t_{1}^{\alpha-1} \leq(\alpha-1)\left|t_{2}-t_{1}\right|
$$

This implies that

$$
\begin{aligned}
& \left|T_{1}(u, v)\left(t_{2}\right)-T_{1}(u, v)\left(t_{1}\right)\right| \\
& \quad \leq M \int_{0}^{1}\left\{\left(\alpha_{1}-1\right)\left[(1-s)^{\alpha_{1}-1}+1\right] \varrho_{1}+\left(\alpha_{1}-1\right)\left[(1-s)^{\alpha_{2}-1}+1\right] \rho_{1}\right\} d s\left|t_{2}-t_{1}\right| \\
& \quad \rightarrow 0 \quad \text { as } t_{2} \rightarrow t_{1} .
\end{aligned}
$$

Besides, we find

$$
\begin{aligned}
\mid D_{t}^{\gamma_{1}} & T_{1}(u, v)\left(t_{2}\right)-D_{t}^{\gamma_{1}} T_{1}(u, v)\left(t_{1}\right) \mid \\
\leq & \left|M \int_{0}^{1}\left(K_{3}\left(t_{2}, s\right)-K_{3}\left(t_{1}, s\right)\right) d s\right|+\left|M \int_{0}^{1}\left(H_{3}\left(t_{2}, s\right)-H_{3}\left(t_{1}, s\right)\right) d s\right| \\
\leq & \frac{M \Gamma\left(\alpha_{1}\right)}{\Gamma\left(\alpha_{1}-\gamma_{1}\right)} \int_{0}^{1}\left\{\left(\alpha_{1}-\gamma_{1}-1\right)\left[(1-s)^{\alpha_{1}-1}+1\right] \varrho_{1}\right. \\
\quad & \left.+\left(\alpha_{1}-\gamma_{1}-1\right)\left[(1-s)^{\alpha_{2}-1}+1\right] \rho_{1}\right\} d s\left|t_{2}-t_{1}\right| \\
\rightarrow & 0 \quad \text { as } t_{2} \rightarrow t_{1} .
\end{aligned}
$$

Therefore $T_{1}$ is equicontinuous for all $(u, v) \in \Omega$, and thus $T_{2}$ is equicontinuous for all $(u, v) \in \Omega$.

As a consequence, the operator $T(u, v)$ is equicontinuous for all $(u, v) \in \Omega$. By the Arzelà-Ascoli theorem the operator $T(u, v)$ is completely continuous.

\section{Existence of monotone iterative positive solutions}

Now, based on Lemmas 2.5-2.7, we will show that there exist positive extremal solutions for BVP (1.1)-(1.2) by the monotone iterative method. 
Theorem 3.1 Assume that $\left(\mathrm{F}_{1}\right)-\left(\mathrm{F}_{4}\right)$ hold. Let $A_{1}, A_{2}$, and $l$ be three positive constants satisfying

$$
\begin{aligned}
& l=\max \left\{A_{1} \int_{0}^{1} \varrho_{1}\left[(1-s)^{\alpha_{1}-1}+1\right] d s+A_{2} \int_{0}^{1}\left[(1-s)^{\alpha_{2}-1}+1\right] \rho_{1} d s,\right. \\
& \left.A_{1} \int_{0}^{1} \rho_{2}\left[(1-s)^{\alpha_{1}-1}+1\right] d s+A_{2} \int_{0}^{1} \varrho_{2}\left[(1-s)^{\alpha_{2}-1}+1\right] d s\right\}
\end{aligned}
$$

(S1) For $t \in[0,1], f_{j}\left(t, x_{1}, x_{2}, x_{3}, x_{4}\right)$ is increasing in $x_{i} \in[0, l](i=1,2,3,4)$ for $j=1,2$;

(S2) $\max _{0 \leq t \leq 1} f_{i}(t, l, l, l, l) \leq A_{i}, f_{i}(t, 0,0,0,0) \not \equiv 0,0 \leq t \leq 1$, for $i=1,2$.

Then BVP (1.1)-(1.2) has positive solutions $\left(u^{*}, v^{*}\right)$ and $\left(w^{*}, z^{*}\right)$ satisfying $0 \leq\left\|\left(u^{*}, v^{*}\right)\right\| \leq l$, and $\lim _{n \rightarrow \infty}\left(u_{n}, v_{n}\right)=\left(u^{*}, v^{*}\right)$,

$$
\left(u_{n}, v_{n}\right)=T\left(u_{n-1}, v_{n-1}\right)=\left(\begin{array}{l}
T_{1}\left(u_{n-1}, v_{n-1}\right) \\
T_{2}\left(u_{n-1}, v_{n-1}\right)
\end{array}\right), \quad n=1,2, \ldots
$$

and

$$
\left(\begin{array}{l}
u_{0}(t) \\
v_{0}(t)
\end{array}\right)=\left(\begin{array}{l}
t^{\alpha_{1}-1} \int_{0}^{1}\left\{A_{1} \varrho_{1}\left[(1-s)^{\alpha_{1}-1}+1\right]+A_{2} \rho_{1}\left[(1-s)^{\alpha_{2}-1}+1\right]\right\} d s \\
t^{\alpha_{2}-1} \int_{0}^{1}\left\{A_{1} \rho_{2}\left[(1-s)^{\alpha_{1}-1}+1\right]+A_{2} \varrho_{2}\left[(1-s)^{\alpha_{2}-1}+1\right]\right\} d s
\end{array}\right)
$$

$0 \leq\left\|\left(w^{*}, z^{*}\right)\right\| \leq l$, and $\lim _{n \rightarrow \infty}\left(w_{n}, z_{n}\right)=\left(w^{*}, z^{*}\right)$,

$$
\left(w_{n}, z_{n}\right)=T\left(w_{n-1}, z_{n-1}\right)=\left(\begin{array}{l}
T_{1}\left(w_{n-1}, z_{n-1}\right) \\
T_{2}\left(w_{n-1}, z_{n-1}\right)
\end{array}\right), \quad n=1,2, \ldots
$$

and

$$
\left(\begin{array}{l}
w_{0}(t) \\
z_{0}(t)
\end{array}\right)=\left(\begin{array}{l}
0 \\
0
\end{array}\right)
$$

Proof Denote $P_{l}=\{(u, v) \in P \mid\|(u, v)\| \leq l\}$, where $l$ is introduced by (3.1). In the following, we first prove that $T: P_{l} \rightarrow P_{l}$. Let $(u, v) \in P_{l}$. Then for $t \in[0,1]$, we have

$$
\begin{array}{lc}
0 \leq u(t) \leq\|u\| \leq l, & 0 \leq\left|D_{t}^{\gamma_{1}} u(t)\right| \leq\|u\| \leq l, \\
0 \leq v(t) \leq\|v\| \leq l, & 0 \leq\left|D_{t}^{\gamma_{2}} v(t)\right| \leq\|v\| \leq l .
\end{array}
$$

So, for $0 \leq t \leq 1, i=1,2$, by (S1) and (S2) we get

$$
0 \leq f_{i}\left(t, u(t), v(t), D_{t}^{\gamma_{1}} u(t), D_{t}^{\gamma_{2}} v(t)\right) \leq \max _{0 \leq t \leq 1}\left\{f_{i}(t, l, l, l, l)\right\} \leq A_{i} .
$$

Consequently, for $t \in[0,1]$, in view of Lemma 2.5 and (3.4), we have

$$
\begin{aligned}
T_{1}(u, v)(t)= & \int_{0}^{1} K_{1}(t, s) f_{1}\left(s, u(s), v(s), D_{t}^{\gamma_{1}} u(s), D_{t}^{\gamma_{2}} v(s)\right) d s \\
& +\int_{0}^{1} H_{1}(t, s) f_{2}\left(s, u(s), v(s), D_{t}^{\gamma_{1}} u(s), D_{t}^{\gamma_{2}} v(s)\right) d s
\end{aligned}
$$




$$
\begin{aligned}
& \leq A_{1} \int_{0}^{1}\left[(1-s)^{\alpha_{1}-1}+1\right] \varrho_{1} d s+A_{2} \int_{0}^{1}\left[(1-s)^{\alpha_{2}-1}+1\right] \rho_{1} d s \leq l \\
&\left|D_{t}^{\gamma_{1}} T_{1}(u, v)(t)\right|=\mid \int_{0}^{1} K_{3}(t, s) f_{1}\left(s, u(s), v(s), D_{t}^{\gamma_{1}} u(s), D_{t}^{\gamma_{2}} v(s)\right) d s \\
&+\int_{0}^{1} H_{3}(t, s) f_{2}\left(s, u(s), v(s), D_{t}^{\gamma_{1}} u(s), D_{t}^{\gamma_{2}} v(s)\right) d s \mid \\
& \leq A_{1} \int_{0}^{1} \frac{\Gamma\left(\alpha_{1}\right)}{\Gamma\left(\alpha_{1}-\gamma_{1}\right)}\left[(1-s)^{\alpha_{1}-1}+1\right] \varrho_{1} d s \\
&+A_{2} \int_{0}^{1} \frac{\Gamma\left(\alpha_{1}\right)}{\Gamma\left(\alpha_{1}-\gamma_{1}\right)}\left[(1-s)^{\alpha_{2}-1}+1\right] \rho_{1} d s \\
& \leq A_{1} \int_{0}^{1}\left[(1-s)^{\alpha_{1}-1}+1\right] \varrho_{1} d s+A_{2} \int_{0}^{1}\left[(1-s)^{\alpha_{2}-1}+1\right] \rho_{1} d s \leq l
\end{aligned}
$$

and, further,

$$
\begin{aligned}
T_{2}(u, v)(t)= & \int_{0}^{1} K_{2}(t, s) f_{2}\left(s, u(s), v(s), D_{t}^{\gamma_{1}} u(s), D_{t}^{\gamma_{2}} v(s)\right) d s \\
& +\int_{0}^{1} H_{2}(t, s) f_{1}\left(s, u(s), v(s), D_{t}^{\gamma_{1}} u(s), D_{t}^{\gamma_{2}} v(s)\right) d s \\
\leq & A_{2} \int_{0}^{1}\left[(1-s)^{\alpha_{2}-1}+1\right] \varrho_{2} d s+A_{1} \int_{0}^{1}\left[(1-s)^{\alpha_{1}-1}+1\right] \rho_{2} d s \leq l, \\
\left|D_{t}^{\gamma_{2}} T_{2}(u, v)(t)\right|= & \mid \int_{0}^{1} K_{4}(t, s) f_{2}\left(s, u(s), v(s), D_{t}^{\gamma_{1}} u(s), D_{t}^{\gamma_{2}} v(s)\right) d s \\
& +\int_{0}^{1} H_{4}(t, s) f_{1}\left(s, u(s), v(s), D_{t}^{\gamma_{1}} u(s), D_{t}^{\gamma_{2}} v(s)\right) d s \mid \\
\leq & A_{2} \int_{0}^{1} \frac{\Gamma\left(\alpha_{2}\right)}{\Gamma\left(\alpha_{2}-\gamma_{2}\right)}\left[(1-s)^{\alpha_{2}-1}+1\right] \varrho_{2} d s \\
& +A_{1} \int_{0}^{1} \frac{\Gamma\left(\alpha_{2}\right)}{\Gamma\left(\alpha_{2}-\gamma_{2}\right)}\left[(1-s)^{\alpha_{1}-1}+1\right] \rho_{2} d s \\
\leq & A_{2} \int_{0}^{1}\left[(1-s)^{\alpha_{2}-1}+1\right] \varrho_{2} d s+A_{1} \int_{0}^{1}\left[(1-s)^{\alpha_{1}-1}+1\right] \rho_{2} d s \leq l .
\end{aligned}
$$

As a result, we obtain

$$
\|T(u, v)\|=\left\{\max _{0 \leq t \leq 1}\left|T_{1} u(t)\right|, \max _{0 \leq t \leq 1}\left|D_{t}^{\gamma_{1}} T_{1} u(t)\right|, \max _{0 \leq t \leq 1}\left|T_{2} v(t)\right|, \max _{0 \leq t \leq 1}\left|D_{t}^{\gamma_{2}} T_{2} v(t)\right|\right\} \leq l,
$$

and thus $T: P_{l} \rightarrow P_{l}$.

According to (3.2) and (3.3), it is obvious that $\left(u_{0}, v_{0}\right),\left(w_{0}, z_{0}\right) \in P_{l}$. Using the completely continuous operator $T$, we define the sequences $\left\{\left(u_{n}, v_{n}\right)\right\}$ and $\left\{\left(w_{n}, z_{n}\right)\right\}$ as $\left(u_{n}, v_{n}\right)=$ $T\left(u_{n-1}, v_{n-1}\right),\left(w_{n}, z_{n}\right)=T\left(w_{n-1}, z_{n-1}\right)$ for $n=1,2, \ldots$. Since $T: P_{l} \rightarrow P_{l}$, we get that $\left(u_{n}, v_{n}\right),\left(w_{n}, z_{n}\right) \in P_{l}$ for $n=1,2, \ldots$

Hence we prove that there exist $\left(u^{*}, v^{*}\right)$ and $\left(w^{*}, z^{*}\right)$ satisfying $\lim _{n \rightarrow \infty}\left(u_{n}, v_{n}\right)=\left(u^{*}, v^{*}\right)$ and $\lim _{n \rightarrow \infty}\left(w_{n}, z_{n}\right)=\left(w^{*}, z^{*}\right)$, which are monotone positive solutions of problem (1.1)(1.2). 
For $t \in[0,1]$, by the definition of the iterative scheme we have

$$
\begin{aligned}
& T_{1}\left(u_{0}, v_{0}\right)(t) \\
& \quad=\int_{0}^{1} K_{1}(t, s) f_{1\left(u_{0}, v_{0}\right)}(s) d s+\int_{0}^{1} H_{1}(t, s) f_{2\left(u_{0}, v_{0}\right)}(s) d s \\
& \quad \leq t^{\alpha_{1}-1} \int_{0}^{1}\left\{A_{1} \varrho_{1}\left[(1-s)^{\alpha_{1}-1}+1\right]+A_{2} \rho_{1}\left[(1-s)^{\alpha_{2}-1}+1\right]\right\} d s \\
& \quad=u_{0}(t)
\end{aligned}
$$

and

$$
\begin{aligned}
& T_{2}\left(u_{0}, v_{0}\right)(t) \\
& \quad=\int_{0}^{1} K_{2}(t, s) f_{2\left(u_{0}, v_{0}\right)}(s) d s+\int_{0}^{1} H_{2}(t, s) f_{1\left(u_{0}, v_{0}\right)}(s) d s \\
& \quad \leq t^{\alpha_{2}-1} \int_{0}^{1}\left\{A_{1} \rho_{2}\left[(1-s)^{\alpha_{1}-1}+1\right]+A_{2} \varrho_{2}\left[(1-s)^{\alpha_{2}-1}+1\right]\right\} d s \\
& \quad=v_{0}(t) .
\end{aligned}
$$

From (3.5) and (3.6) we get

$$
\left(\begin{array}{l}
u_{1}(t) \\
v_{1}(t)
\end{array}\right)=\left(\begin{array}{l}
T_{1}\left(u_{0}, v_{0}\right)(t) \\
T_{2}\left(u_{0}, v_{0}\right)(t)
\end{array}\right) \leq\left(\begin{array}{l}
u_{0}(t) \\
v_{0}(t)
\end{array}\right)
$$

Next, we discuss the monotonicity of the fractional derivative of $(u, v)$. From (3.2) we obtain

$$
\begin{aligned}
& D_{t}^{\gamma_{1}} u_{0}(t)=\int_{0}^{1} \frac{\Gamma\left(\alpha_{1}\right) t^{\alpha_{1}-\gamma_{1}-1}}{\Gamma\left(\alpha_{1}-\gamma_{1}\right)}\left\{A_{1} \varrho_{1}\left[(1-s)^{\alpha_{1}-1}+1\right]+A_{2} \rho_{1}\left[(1-s)^{\alpha_{2}-1}+1\right]\right\} d s \geq 0, \\
& D_{t}^{\gamma_{2}} v_{0}(t)=\int_{0}^{1} \frac{\Gamma\left(\alpha_{2}\right) t^{\alpha_{2}-\gamma_{2}-1}}{\Gamma\left(\alpha_{2}-\gamma_{2}\right)}\left\{A_{1} \rho_{2}\left[(1-s)^{\alpha_{1}-1}+1\right]+A_{2} \varrho_{2}\left[(1-s)^{\alpha_{2}-1}+1\right]\right\} d s \geq 0 .
\end{aligned}
$$

Hence we have

$$
\begin{aligned}
\left|D_{t}^{\gamma_{1}} u_{1}(t)\right|=\left|D_{t}^{\gamma_{1}} T_{1}\left(u_{0}, v_{0}\right)(t)\right| & \mid \int_{0}^{1} K_{3}(t, s) f_{1\left(u_{0}, v_{0}\right)}(s) d s+\int_{0}^{1} H_{3}(t, s) f_{2}\left(u_{0}, v_{0}\right) \\
= & (s) d s \mid \\
\leq & A_{1} \int_{0}^{1} \frac{\Gamma\left(\alpha_{1}\right) t^{\alpha_{1}-\gamma_{1}-1}}{\Gamma\left(\alpha_{1}-\gamma_{1}\right)}\left[(1-s)^{\alpha_{1}-1}+1\right] \varrho_{1} d s \\
& +A_{2} \int_{0}^{1} \frac{\Gamma\left(\alpha_{1}\right) t^{\alpha_{1}-\gamma_{1}-1}}{\Gamma\left(\alpha_{1}-\gamma_{1}\right)}\left[(1-s)^{\alpha_{2}-1}+1\right] \rho_{1} d s \\
= & \left|D_{t}^{\gamma_{1}} u_{0}(t)\right|
\end{aligned}
$$

and

$$
\left|D_{t}^{\gamma_{2}} v_{1}(t)\right|=\left|D_{t}^{\gamma_{2}} T_{2}\left(u_{0}, v_{0}\right)(t)\right|
$$




$$
\begin{aligned}
= & \left|\int_{0}^{1} K_{4}(t, s) f_{2\left(u_{0}, v_{0}\right)}(s) d s+\int_{0}^{1} H_{4}(t, s) f_{1\left(u_{0}, v_{0}\right)}(s) d s\right| \\
\leq & A_{2} \int_{0}^{1} \frac{\Gamma\left(\alpha_{2}\right) t^{\alpha_{2}-\gamma_{2}-1}}{\Gamma\left(\alpha_{2}-\gamma_{2}\right)}\left[(1-s)^{\alpha_{2}-1}+1\right] \varrho_{2} d s \\
& +A_{1} \int_{0}^{1} \frac{\Gamma\left(\alpha_{2}\right) t^{\alpha_{2}-\gamma_{2}-1}}{\Gamma\left(\alpha_{2}-\gamma_{2}\right)}\left[(1-s)^{\alpha_{1}-1}+1\right] \rho_{2} d s \\
= & \left|D_{t}^{\gamma_{2}} v_{0}(t)\right| .
\end{aligned}
$$

Combining (3.8) and (3.9), we easily to see that

$$
\left(\begin{array}{l}
\left|D_{t}^{\gamma_{1}} u_{1}(t)\right| \\
\left|D_{t}^{\gamma_{2}} v_{1}(t)\right|
\end{array}\right)=\left(\begin{array}{l}
\left|D_{t}^{\gamma_{1}} T_{1}\left(u_{0}, v_{0}\right)(t)\right| \\
\left|D_{t}^{\gamma_{2}} T_{2}\left(u_{0}, v_{0}\right)(t)\right|
\end{array}\right) \leq\left(\begin{array}{c}
\left|D_{t}^{\gamma_{1}} u_{0}(t)\right| \\
\left|D_{t}^{\gamma_{2}} v_{0}(t)\right|
\end{array}\right)
$$

Thus, for $0 \leq t \leq 1$, from (3.7), (3.10), and (S1) we do the second iteration

$$
\begin{aligned}
& \left(\begin{array}{l}
u_{2}(t) \\
v_{2}(t)
\end{array}\right)=\left(\begin{array}{l}
T_{1}\left(u_{1}, v_{1}\right)(t) \\
T_{2}\left(u_{1}, v_{1}\right)(t)
\end{array}\right) \leq\left(\begin{array}{l}
T_{1}\left(u_{0}, v_{0}\right)(t) \\
T_{2}\left(u_{0}, v_{0}\right)(t)
\end{array}\right)=\left(\begin{array}{l}
u_{1}(t) \\
v_{1}(t)
\end{array}\right), \\
& \left(\begin{array}{l}
\left|D_{t}^{\gamma_{1}} u_{2}(t)\right| \\
\left|D_{t}^{\gamma_{2}} v_{2}(t)\right|
\end{array}\right)=\left(\begin{array}{l}
\left|D_{t}^{\gamma_{1}} T_{1}\left(u_{1}, v_{1}\right)(t)\right| \\
\left|D_{t}^{\gamma_{2}} T_{2}\left(u_{1}, v_{1}\right)(t)\right|
\end{array}\right) \leq\left(\begin{array}{l}
\left|D_{t}^{\gamma_{1}} T_{1}\left(u_{0}, v_{0}\right)(t)\right| \\
\left|D_{t}^{\gamma_{2}} T_{2}\left(u_{0}, v_{0}\right)(t)\right|
\end{array}\right)=\left(\begin{array}{l}
\left|D_{t}^{\gamma_{1}} u_{1}(t)\right| \\
\left|D_{t}^{\gamma_{2}} v_{1}(t)\right|
\end{array}\right) .
\end{aligned}
$$

By induction, for $n=0,1,2, \ldots$, we have

$$
\left(\begin{array}{l}
u_{n+1}(t) \\
v_{n+1}(t)
\end{array}\right) \leq\left(\begin{array}{l}
u_{n}(t) \\
v_{n}(t)
\end{array}\right), \quad\left(\begin{array}{l}
\left|D_{t}^{\gamma_{1}} u_{n+1}(t)\right| \\
\left|D_{t}^{\gamma_{2}} v_{n+1}(t)\right|
\end{array}\right) \leq\left(\begin{array}{l}
\left|D_{t}^{\gamma_{1}} u_{n}(t)\right| \\
\left|D_{t}^{\gamma_{2}} v_{n}(t)\right|
\end{array}\right) \quad \text { for } 0 \leq t \leq 1
$$

So we assert that $\left(u_{n}, v_{n}\right) \rightarrow\left(u^{*}, v^{*}\right)$ and $T\left(u^{*}, v^{*}\right)=\left(u^{*}, v^{*}\right)$, since $T$ is completely continuous and $\left(u_{n+1}, v_{n+1}\right)=T\left(u_{n}, v_{n}\right)$.

For the sequence $\left\{\left(w_{n}, z_{n}\right)\right\}_{n=1}^{\infty}$, we apply a similar argument. For $t \in[0,1]$, we have

$$
\begin{aligned}
\left(\begin{array}{l}
w_{1}(t) \\
z_{1}(t)
\end{array}\right)= & \left(\begin{array}{l}
T_{1}\left(w_{0}, z_{0}\right)(t) \\
T_{2}\left(w_{0}, z_{0}\right)(t)
\end{array}\right) \\
= & \left(\begin{array}{l}
\int_{0}^{1} K_{1}(t, s) f_{1\left(w_{0}, z_{0}\right)}(s) d s+\int_{0}^{1} H_{1}(t, s) f_{2\left(w_{0}, z_{0}\right)}(s) d s \\
\int_{0}^{1} K_{2}(t, s) f_{2\left(w_{0}, z_{0}\right)}(s) d s+\int_{0}^{1} H_{2}(t, s) f_{1\left(w_{0}, z_{0}\right)}(s) d s
\end{array}\right) \\
\geq\left(\begin{array}{l}
0 \\
0
\end{array}\right) & =\left(\begin{array}{l}
w_{0}(t) \\
z_{0}(t)
\end{array}\right) ; \\
\left(\begin{array}{l}
\left|D_{t}^{\gamma_{1}} w_{1}(t)\right| \\
\left|D_{t}^{\gamma_{2}} z_{1}(t)\right|
\end{array}\right) & =\left(\begin{array}{l}
\left|D_{t}^{\gamma_{1}} T_{1}\left(w_{0}, z_{0}\right)(t)\right| \\
\left|D_{t}^{\gamma_{2}} T_{2}\left(w_{0}, z_{0}\right)(t)\right|
\end{array}\right) \\
& =\left(\begin{array}{l}
\left|\int_{0}^{1} K_{3}(t, s) f_{1\left(w_{0}, z_{0}\right)}(s) d s+\int_{0}^{1} H_{3}(t, s) f_{2\left(w_{0}, z_{0}\right)}(s) d s\right| \\
\left|\int_{0}^{1} K_{4}(t, s) f_{2\left(w_{0}, z_{0}\right)}(s) d s+\int_{0}^{1} H_{4}(t, s) f_{1\left(w_{0}, z_{0}\right)}(s) d s\right|
\end{array}\right) \\
\geq & \left(\begin{array}{l}
0 \\
0
\end{array}\right)=\left(\begin{array}{c}
\left|D_{t}^{\gamma_{1}} w_{0}(t)\right| \\
\left|D_{t}^{\gamma_{2}} z_{0}(t)\right|
\end{array}\right) .
\end{aligned}
$$


To summarize, for $0 \leq t \leq 1$, we have

$$
\begin{aligned}
& \left(\begin{array}{l}
w_{2}(t) \\
z_{2}(t)
\end{array}\right)=\left(\begin{array}{l}
T_{1}\left(w_{1}, z_{1}\right)(t) \\
T_{2}\left(w_{1}, z_{1}\right)(t)
\end{array}\right) \geq\left(\begin{array}{l}
T_{1}\left(w_{0}, z_{0}\right)(t) \\
T_{2}\left(w_{0}, z_{0}\right)(t)
\end{array}\right)=\left(\begin{array}{c}
w_{1}(t) \\
z_{1}(t)
\end{array}\right), \\
& \left(\begin{array}{c}
\left|D_{t}^{\gamma_{1}} w_{2}(t)\right| \\
\left|D_{t}^{\gamma_{2}} z_{2}(t)\right|
\end{array}\right)=\left(\begin{array}{c}
\left|D_{t}^{\gamma_{1}} T_{1}\left(w_{1}, z_{1}\right)(t)\right| \\
\left|D_{t}^{\gamma_{2}} T_{2}\left(w_{1}, z_{1}\right)(t)\right|
\end{array}\right) \geq\left(\begin{array}{c}
\left|D_{t}^{\gamma_{1}} T_{1}\left(w_{0}, z_{0}\right)(t)\right| \\
\left|D_{t}^{\gamma_{2}} T_{2}\left(w_{0}, z_{0}\right)(t)\right|
\end{array}\right)=\left(\begin{array}{c}
\left|D_{t}^{\gamma_{1}} w_{1}(t)\right| \\
\left|D_{t}^{\gamma_{2}} z_{1}(t)\right|
\end{array}\right) .
\end{aligned}
$$

Analogously, for $n=1,2, \ldots$, we have

$$
\left(\begin{array}{c}
w_{n+1}(t) \\
z_{n+1}(t)
\end{array}\right) \geq\left(\begin{array}{c}
w_{n}(t) \\
z_{n}(t)
\end{array}\right), \quad\left(\begin{array}{c}
\left|D_{t}^{\gamma_{1}} w_{n+1}(t)\right| \\
\left|D_{t}^{\gamma_{2}} z_{n+1}(t)\right|
\end{array}\right) \geq\left(\begin{array}{c}
\left|D_{t}^{\gamma_{1}} w_{n}(t)\right| \\
\left|D_{t}^{\gamma_{2}} z_{n}(t)\right|
\end{array}\right) \quad \text { for } 0 \leq t \leq 1
$$

So we can assert that $\left(w_{n}, z_{n}\right) \rightarrow\left(w^{*}, z^{*}\right)$ and $T\left(w^{*}, z^{*}\right)=\left(w^{*}, z^{*}\right)$, since $T$ is completely continuous and $\left(w_{n+1}, z_{n+1}\right)=T\left(w_{n}, z_{n}\right)$.

Consequently, there exist $\left(u^{*}, v^{*}\right)$ and $\left(w^{*}, z^{*}\right)$ in $P_{l}$ that are nonnegative extremal solutions of BVP (1.1)-(1.2). From (S2) it is obvious that $\left(u^{*}, v^{*}\right)(t)>0$ and $\left(w^{*}, z^{*}\right)(t)>0$ for $t \in[0,1]$, since zero is not a solution of problem (1.1). The proof is completed.

Example 3.1 For $t \in[0,1]$, consider the following fractional differential system:

$$
\left\{\begin{array}{l}
D_{t}^{2.5} u(t)+\left(\frac{1}{2} t^{2}+\frac{1}{10} u(t)+\frac{1}{32} \sin (v(t))+\frac{1}{50} D_{t}^{0.3} u(t)+\frac{1}{D_{t}^{0.2} v(t)-\frac{1}{2}}+2\right)=0 \\
D_{t}^{2.5} v(t)+\left(\frac{1}{3} t+\frac{1}{4 \pi} \sin (2 \pi u(t))+\frac{1}{18} v(t)+\frac{1}{5}\left(D_{t}^{0.3} u(t)\right)^{2}+D_{t}^{0.2} v(t)+\frac{10}{11}\right)=0
\end{array}\right.
$$

with the coupled integral and discrete mixed boundary conditions

$$
\begin{cases}u(0)=u^{\prime}(0)=0, & u(1)=\sum_{i=1}^{2} \lambda_{1 i} I_{t}^{1.5} v\left(\xi_{i}\right)+\sum_{i=1}^{2} b_{i} v\left(\eta_{i}\right) \\ v(0)=v^{\prime}(0)=0, & v(1)=\sum_{i=1}^{2} \lambda_{2 i} I_{t}^{1.2} u\left(\xi_{i}\right)+\sum_{i=1}^{2} b_{i} u\left(\eta_{i}\right)\end{cases}
$$

In this model, we set

$$
\begin{aligned}
& \lambda_{11}=\frac{1}{2}, \quad \lambda_{21}=\frac{2}{7}, \quad \xi_{1}=\frac{1}{4}, \quad b_{1}=\frac{1}{3}, \quad \eta_{1}=\frac{1}{3} \text {, } \\
& \lambda_{12}=\frac{1}{4}, \quad \lambda_{22}=\frac{3}{7}, \quad \xi_{2}=\frac{3}{4}, \quad b_{2}=\frac{2}{3}, \quad \eta_{2}=\frac{2}{3} \text {. }
\end{aligned}
$$

It is obvious that $\left(\mathrm{F}_{1}\right)$ and $\left(\mathrm{F}_{2}\right)$ hold. By calculation we get

$$
\begin{array}{ll}
l_{1}=0.4920, & l_{2}=0.4521, \\
\varrho_{1}=0.9675, & \varrho_{2}=0.9675, \\
\rho_{1}=0.4374, & \rho_{2}=0.4760 .
\end{array}
$$

Setting $A_{1}=A_{2}=5$, we get $l=\max \left\{A_{1} \int_{0}^{1} \varrho_{1}\left[(1-s)^{\alpha_{1}-1}+1\right] d s+A_{2} \int_{0}^{1}\left[(1-s)^{\alpha_{2}-1}+\right.\right.$ $\left.1] \rho_{1} d s, A_{1} \int_{0}^{1} \rho_{2}\left[(1-s)^{\alpha_{1}-1}+1\right] d s+A_{2} \int_{0}^{1} \varrho_{2}\left[(1-s)^{\alpha_{2}-1}+1\right] d s\right\}$. Then all the hypotheses of Theorem 3.1 are satisfied with $l=11$. Hence, BVP (3.11)-(3.12) has monotone positive 
solutions $\left(u^{*}, v^{*}\right)$ and $\left(w^{*}, z^{*}\right)$, which satisfy, for $t \in[0,1]$,

$$
\begin{aligned}
\left(\begin{array}{l}
u_{0}(t) \\
v_{0}(t)
\end{array}\right) & =\left(\begin{array}{c}
t^{\alpha_{1}-1}\left\{A_{1} \int_{0}^{1} \varrho_{1}\left[(1-s)^{\alpha_{1}-1}+1\right] d s+A_{2} \int_{0}^{1}\left[(1-s)^{\alpha_{2}-1}+1\right] \rho_{1} d s\right\} \\
t^{\alpha_{2}-1}\left\{A_{1} \int_{0}^{1} \rho_{2}\left[(1-s)^{\alpha_{1}-1}+1\right] d s+A_{2} \int_{0}^{1}\left[(1-s)^{\alpha_{2}-1}+1\right] \varrho_{2} d s\right\}
\end{array}\right) \\
& =\left(\begin{array}{c}
9.8343 t^{1.5} \\
10.1045 t^{1.5}
\end{array}\right)
\end{aligned}
$$

and

$$
\left(\begin{array}{l}
w_{0}(t) \\
z_{0}(t)
\end{array}\right)=\left(\begin{array}{l}
0 \\
0
\end{array}\right)
$$

For $n=1,2, \ldots$, the two iterative schemes are

$$
\begin{aligned}
& \left(\begin{array}{l}
u_{0}(t) \\
v_{0}(t)
\end{array}\right)=\left(\begin{array}{c}
9.8343 t^{1.5} \\
10.1045 t^{1.5}
\end{array}\right), \quad \ldots, \\
& \left(\begin{array}{l}
u_{n+1}(t) \\
v_{n+1}(t)
\end{array}\right)=\left(\begin{array}{l}
T_{1}\left(u_{n}, v_{n}\right)(t) \\
T_{2}\left(u_{n}, v_{n}\right)(t)
\end{array}\right)=\left(\begin{array}{l}
\int_{0}^{1} K_{1}(t, s) f_{1\left(u_{n}, v_{n}\right)}(s) d s+\int_{0}^{1} H_{1}(t, s) f_{2\left(u_{n}, v_{n}\right)}(s) d s \\
\int_{0}^{1} K_{2}(t, s) f_{2\left(u_{n}, v_{n}\right)}(s) d s+\int_{0}^{1} H_{2}(t, s) f_{1}\left(u_{n}, v_{n}\right)
\end{array}\right)
\end{aligned}
$$

where

$$
\begin{aligned}
& \int_{0}^{1} K_{1}(t, s) f_{1\left(u_{n}, v_{n}\right)}(s) d s+\int_{0}^{1} H_{1}(t, s) f_{2\left(u_{n}, v_{n}\right)}(s) d s \\
& =\frac{1}{\Gamma(2.5)} \int_{0}^{1}\left\{t^{1.5}(1-s)^{1.5}+\frac{l_{2} t^{1.5}}{1-l_{1} l_{2}}\left[\frac{2}{7 \Gamma(1.2)} \int_{0}^{\frac{1}{4}}\left(\frac{1}{4}-\tau\right)^{0.2} \tau^{1.5}(1-s)^{1.5} d \tau\right.\right. \\
& -\frac{2}{7 \Gamma(1.2)} \int_{s}^{\frac{1}{4}}\left(\frac{1}{4}-\tau\right)^{0.2}(\tau-s)^{1.5} d \tau+\frac{3}{7 \Gamma(1.2)} \int_{0}^{\frac{3}{4}}\left(\frac{3}{4}-\tau\right)^{0.2} \tau^{1.5}(1-s)^{1.5} d \tau \\
& \left.\left.-\frac{3}{7 \Gamma(1.2)} \int_{s}^{\frac{3}{4}}\left(\frac{3}{4}-\tau\right)^{0.2}(\tau-s)^{1.5} d \tau+\frac{1}{3} \cdot \frac{1}{3}^{1.5}(1-s)^{1.5}+\frac{2}{3} \cdot \frac{2}{3}^{1.5}(1-s)^{1.5}\right]\right\} \\
& \times\left(\frac{1}{2} s^{2}+\frac{1}{10} u_{n}(s)+\frac{1}{32} \sin \left(v_{n}(s)\right)+\frac{1}{50} D_{t}^{0.3} u_{n}(s)+\frac{1}{D_{t}^{0.2} v_{n}(s)-\frac{1}{2}}+2\right) d s \\
& -\frac{1}{\Gamma(2.5)} \int_{0}^{t}\left\{(t-s)^{1.5}+\frac{l_{2} t^{1.5}}{1-l_{1} l_{2}}\left[\frac{1}{3}\left(\frac{1}{3}-s\right)^{1.5}+\frac{2}{3}\left(\frac{2}{3}-s\right)^{1.5}\right]\right\}\left(\frac{1}{2} s^{2}+\frac{1}{10} u_{n}(s)\right. \\
& \left.+\frac{1}{32} \sin \left(v_{n}(s)\right)+\frac{1}{50} D_{t}^{0.3} u_{n}(s)+\frac{1}{D_{t}^{0.2} v_{n}(s)-\frac{1}{2}}+2\right) d s \\
& +\frac{1}{\Gamma(2.5)} \int_{0}^{1} \frac{t^{1.5}}{1-l_{1} l_{2}}\left[\frac{1}{2 \Gamma(1.5)} \int_{0}^{\frac{1}{4}}\left(\frac{1}{4}-\tau\right)^{0.5} \tau^{1.5}(1-s)^{1.5} d \tau\right. \\
& -\frac{1}{2 \Gamma(1.5)} \int_{s}^{\frac{1}{4}}\left(\frac{1}{4}-\tau\right)^{0.5}(\tau-s)^{1.5} d \tau+\frac{1}{4 \Gamma(1.5)} \int_{0}^{\frac{3}{4}}\left(\frac{3}{4}-\tau\right)^{0.5} \tau^{1.5}(1-s)^{1.5} d \tau \\
& \left.-\frac{1}{4 \Gamma(1.5)} \int_{s}^{\frac{3}{4}}\left(\frac{3}{4}-\tau\right)^{0.5}(\tau-s)^{1.5} d \tau+\frac{1}{3} \cdot \frac{1}{3}^{1.5}(1-s)^{1.5}+\frac{2}{3} \cdot \frac{2}{3}^{1.5}(1-s)^{1.5}\right] \\
& \times\left(\frac{1}{3} s+\frac{1}{4 \pi} \sin \left(2 \pi u_{n}(s)\right)+\frac{1}{18} v_{n}(t)+\frac{1}{5}\left(D_{t}^{0.3} u_{n}(t)\right)^{2}+D_{t}^{0.2} v_{n}(t)+\frac{10}{11}\right) d s
\end{aligned}
$$


Zhao et al. Advances in Difference Equations

(2019) 2019:389

Page 20 of 23

$$
\begin{aligned}
& -\frac{1}{\Gamma(2.5)} \int_{0}^{t} \frac{t^{1.5}}{1-l_{1} l_{2}}\left[\frac{1}{3}\left(\frac{1}{3}-s\right)^{1.5}+\frac{2}{3}\left(\frac{2}{3}-s\right)^{1.5}\right] \\
& \times\left(\frac{1}{3} s+\frac{1}{4 \pi} \sin \left(2 \pi u_{n}(s)\right)+\frac{1}{18} v_{n}(t)\right. \\
& \left.+\frac{1}{5}\left(D_{t}^{0.3} u_{n}(t)\right)^{2}+D_{t}^{0.2} v_{n}(t)+\frac{10}{11}\right) d s, \\
& \int_{0}^{1} K_{2}(t, s) f_{2\left(u_{n}, v_{n}\right)}(s) d s+\int_{0}^{1} H_{2}(t, s) f_{1\left(u_{n}, v_{n}\right)}(s) d s \\
& =\frac{1}{\Gamma(2.5)} \int_{0}^{1}\left\{t^{1.5}(1-s)^{1.5}+\frac{l_{1} t^{1.5}}{1-l_{1} l_{2}}\left[\frac{1}{2 \Gamma(1.5)} \int_{0}^{\frac{1}{4}}\left(\frac{1}{4}-\tau\right)^{0.5} \tau^{1.5}(1-s)^{1.5} d \tau\right.\right. \\
& -\frac{1}{2 \Gamma(1.5)} \int_{s}^{\frac{1}{4}}\left(\frac{1}{4}-\tau\right)^{0.5}(\tau-s)^{1.5} d \tau+\frac{1}{4 \Gamma(1.5)} \int_{0}^{\frac{3}{4}}\left(\frac{3}{4}-\tau\right)^{0.5} \tau^{1.5}(1-s)^{1.5} d \tau \\
& \left.\left.-\frac{1}{4 \Gamma(1.5)} \int_{s}^{\frac{3}{4}}\left(\frac{3}{4}-\tau\right)^{0.5}(\tau-s)^{1.5} d \tau+\frac{1}{3} \cdot \frac{1}{3}^{1.5}(1-s)^{1.5}+\frac{2}{3} \cdot \frac{2}{3}^{1.5}(1-s)^{1.5}\right]\right\} \\
& \times\left(\frac{1}{3} s+\frac{1}{4 \pi} \sin \left(2 \pi u_{n}(s)\right)+\frac{1}{18} v_{n}(t)+\frac{1}{5}\left(D_{t}^{0.3} u_{n}(t)\right)^{2}+D_{t}^{0.2} v_{n}(t)+\frac{10}{11}\right) d s \\
& -\frac{1}{\Gamma(2.5)} \int_{0}^{t}\left\{(t-s)^{1.5}+\frac{l_{1} t^{1.5}}{1-l_{1} l_{2}}\left[\frac{1}{3}\left(\frac{1}{3}-s\right)^{1.5}+\frac{2}{3}\left(\frac{2}{3}-s\right)^{1.5}\right]\right\} \\
& \times\left(\frac{1}{3} s+\frac{1}{4 \pi} \sin \left(2 \pi u_{n}(s)\right)\right. \\
& \left.+\frac{1}{18} v_{n}(t)+\frac{1}{5}\left(D_{t}^{0.3} u_{n}(t)\right)^{2}+D_{t}^{0.2} v_{n}(t)+\frac{10}{11}\right) d s \\
& +\frac{1}{\Gamma(2.5)} \int_{0}^{1} \frac{t^{1.5}}{1-l_{1} l_{2}}\left[\frac{2}{7 \Gamma(1.2)} \int_{0}^{\frac{1}{4}}\left(\frac{1}{4}-\tau\right)^{0.2} \tau^{1.5}(1-s)^{1.5} d \tau\right. \\
& -\frac{2}{7 \Gamma(1.2)} \int_{s}^{\frac{1}{4}}\left(\frac{1}{4}-\tau\right)^{0.2}(\tau-s)^{1.5} d \tau+\frac{3}{7 \Gamma(1.2)} \int_{0}^{\frac{3}{4}}\left(\frac{3}{4}-\tau\right)^{0.2} \tau^{1.5}(1-s)^{1.5} d \tau \\
& \left.-\frac{3}{7 \Gamma(1.2)} \int_{s}^{\frac{3}{4}}\left(\frac{3}{4}-\tau\right)^{0.2}(\tau-s)^{1.5} d \tau+\frac{1}{3} \cdot \frac{1}{3}^{1.5}(1-s)^{1.5}+\frac{2}{3} \cdot \frac{2}{3}^{1.5}(1-s)^{1.5}\right] \\
& \times\left(\frac{1}{2} s^{2}+\frac{1}{10} u_{n}(s)+\frac{1}{32} \sin \left(v_{n}(s)\right)+\frac{1}{50} D_{t}^{0.3} u_{n}(s)+\frac{1}{D_{t}^{0.2} v_{n}(s)-\frac{1}{2}}+2\right) d s \\
& -\frac{1}{\Gamma(2.5)} \int_{0}^{t} \frac{t^{1.5}}{1-l_{1} l_{2}}\left[\frac{1}{3}\left(\frac{1}{3}-s\right)^{1.5}+\frac{2}{3}\left(\frac{2}{3}-s\right)^{1.5}\right] \\
& \times\left(\frac{1}{2} s^{2}+\frac{1}{10} u_{n}(s)+\frac{1}{32} \sin \left(v_{n}(s)\right)\right. \\
& \left.+\frac{1}{50} D_{t}^{0.3} u_{n}(s)+\frac{1}{D_{t}^{0.2} v_{n}(s)-\frac{1}{2}}+2\right) d s,
\end{aligned}
$$

and

$$
\left(\begin{array}{l}
w_{0}(t) \\
z_{0}(t)
\end{array}\right)=\left(\begin{array}{l}
0 \\
0
\end{array}\right), \quad \ldots,
$$


Zhao et al. Advances in Difference Equations

(2019) 2019:389

Page 21 of 23

$$
\left(\begin{array}{c}
w_{n+1}(t) \\
z_{n+1}(t)
\end{array}\right)=\left(\begin{array}{l}
\int_{0}^{1} K_{1}(t, s) f_{1\left(w_{n}, z_{n}\right)}(s) d s+\int_{0}^{1} H_{1}(t, s) f_{2\left(w_{n}, z_{n}\right)}(s) d s \\
\int_{0}^{1} K_{2}(t, s) f_{2\left(w_{n}, z_{n}\right)}(s) d s+\int_{0}^{1} H_{2}(t, s) f_{1\left(w_{n}, z_{n}\right)}(s) d s
\end{array}\right)
$$

where

$$
\begin{aligned}
& \int_{0}^{1} K_{1}(t, s) f_{1\left(w_{n}, z_{n}\right)}(s) d s+\int_{0}^{1} H_{1}(t, s) f_{2\left(w_{n}, z_{n}\right)}(s) d s \\
& =\frac{1}{\Gamma(2.5)} \int_{0}^{1}\left\{t^{1.5}(1-s)^{1.5}+\frac{l_{2} t^{1.5}}{1-l_{1} l_{2}}\left[\frac{2}{7 \Gamma(1.2)} \int_{0}^{\frac{1}{4}}\left(\frac{1}{4}-\tau\right)^{0.2} \tau^{1.5}(1-s)^{1.5} d \tau\right.\right. \\
& -\frac{2}{7 \Gamma(1.2)} \int_{s}^{\frac{1}{4}}\left(\frac{1}{4}-\tau\right)^{0.2}(\tau-s)^{1.5} d \tau+\frac{3}{7 \Gamma(1.2)} \int_{0}^{\frac{3}{4}}\left(\frac{3}{4}-\tau\right)^{0.2} \tau^{1.5}(1-s)^{1.5} d \tau \\
& \left.\left.-\frac{3}{7 \Gamma(1.2)} \int_{s}^{\frac{3}{4}}\left(\frac{3}{4}-\tau\right)^{0.2}(\tau-s)^{1.5} d \tau+\frac{1}{3} \cdot \frac{1}{3}^{1.5}(1-s)^{1.5}+\frac{2}{3} \cdot \frac{2}{3}^{1.5}(1-s)^{1.5}\right]\right\} \\
& \times\left(\frac{1}{2} s^{2}+\frac{1}{10} w_{n}(s)+\frac{1}{32} \sin \left(z_{n}(s)\right)+\frac{1}{50} D_{t}^{0.3} w_{n}(s)+\frac{1}{D_{t}^{0.2} z_{n}(s)-\frac{1}{2}}+2\right) d s \\
& -\frac{1}{\Gamma(2.5)} \int_{0}^{t}\left\{(t-s)^{1.5}+\frac{l_{2} t^{1.5}}{1-l_{1} l_{2}}\left[\frac{1}{3}\left(\frac{1}{3}-s\right)^{1.5}+\frac{2}{3}\left(\frac{2}{3}-s\right)^{1.5}\right]\right\}\left(\frac{1}{2} s^{2}+\frac{1}{10} w_{n}(s)\right. \\
& \left.+\frac{1}{32} \sin \left(z_{n}(s)\right)+\frac{1}{50} D_{t}^{0.3} w_{n}(s)+\frac{1}{D_{t}^{0.2} z_{n}(s)-\frac{1}{2}}+2\right) d s \\
& +\frac{1}{\Gamma(2.5)} \int_{0}^{1} \frac{t^{1.5}}{1-l_{1} l_{2}}\left[\frac{1}{2 \Gamma(1.5)} \int_{0}^{\frac{1}{4}}\left(\frac{1}{4}-\tau\right)^{0.5} \tau^{1.5}(1-s)^{1.5} d \tau\right. \\
& -\frac{1}{2 \Gamma(1.5)} \int_{s}^{\frac{1}{4}}\left(\frac{1}{4}-\tau\right)^{0.5}(\tau-s)^{1.5} d \tau+\frac{1}{4 \Gamma(1.5)} \int_{0}^{\frac{3}{4}}\left(\frac{3}{4}-\tau\right)^{0.5} \tau^{1.5}(1-s)^{1.5} d \tau \\
& \left.-\frac{1}{4 \Gamma(1.5)} \int_{s}^{\frac{3}{4}}\left(\frac{3}{4}-\tau\right)^{0.5}(\tau-s)^{1.5} d \tau+\frac{1}{3} \cdot \frac{1}{3}^{1.5}(1-s)^{1.5}+\frac{2}{3} \cdot \frac{2}{3}^{1.5}(1-s)^{1.5}\right] \\
& \times\left(\frac{1}{3} s+\frac{1}{4 \pi} \sin \left(2 \pi w_{n}(s)\right)+\frac{1}{18} z_{n}(t)+\frac{1}{5}\left(D_{t}^{0.3} w_{n}(t)\right)^{2}+D_{t}^{0.2} z_{n}(t)+\frac{10}{11}\right) d s \\
& -\frac{1}{\Gamma(2.5)} \int_{0}^{t} \frac{t^{1.5}}{1-l_{1} l_{2}}\left[\frac{1}{3}\left(\frac{1}{3}-s\right)^{1.5}+\frac{2}{3}\left(\frac{2}{3}-s\right)^{1.5}\right] \\
& \times\left(\frac{1}{3} s+\frac{1}{4 \pi} \sin \left(2 \pi w_{n}(s)\right)+\frac{1}{18} z_{n}(t)\right. \\
& \left.+\frac{1}{5}\left(D_{t}^{0.3} w_{n}(t)\right)^{2}+D_{t}^{0.2} z_{n}(t)+\frac{10}{11}\right) d s \\
& \int_{0}^{1} K_{2}(t, s) f_{2\left(w_{n}, z_{n}\right)}(s) d s+\int_{0}^{1} H_{2}(t, s) f_{1\left(w_{n}, z_{n}\right)}(s) d s \\
& =\frac{1}{\Gamma(2.5)} \int_{0}^{1}\left\{t^{1.5}(1-s)^{1.5}+\frac{l_{1} t^{1.5}}{1-l_{1} l_{2}}\left[\frac{1}{2 \Gamma(1.5)} \int_{0}^{\frac{1}{4}}\left(\frac{1}{4}-\tau\right)^{0.5} \tau^{1.5}(1-s)^{1.5} d \tau\right.\right. \\
& -\frac{1}{2 \Gamma(1.5)} \int_{s}^{\frac{1}{4}}\left(\frac{1}{4}-\tau\right)^{0.5}(\tau-s)^{1.5} d \tau+\frac{1}{4 \Gamma(1.5)} \int_{0}^{\frac{3}{4}}\left(\frac{3}{4}-\tau\right)^{0.5} \tau^{1.5}(1-s)^{1.5} d \tau \\
& \left.\left.-\frac{1}{4 \Gamma(1.5)} \int_{s}^{\frac{3}{4}}\left(\frac{3}{4}-\tau\right)^{0.5}(\tau-s)^{1.5} d \tau+\frac{1}{3} \cdot \frac{1}{3}^{1.5}(1-s)^{1.5}+\frac{2}{3} \cdot \frac{2}{3}^{1.5}(1-s)^{1.5}\right]\right\}
\end{aligned}
$$




$$
\begin{aligned}
& \times\left(\frac{1}{3} s+\frac{1}{4 \pi} \sin \left(2 \pi w_{n}(s)\right)+\frac{1}{18} z_{n}(t)+\frac{1}{5}\left(D_{t}^{0.3} w_{n}(t)\right)^{2}+D_{t}^{0.2} z_{n}(t)+\frac{10}{11}\right) d s \\
& -\frac{1}{\Gamma(2.5)} \int_{0}^{t}\left\{(t-s)^{1.5}+\frac{l_{1} t^{1.5}}{1-l_{1} l_{2}}\left[\frac{1}{3}\left(\frac{1}{3}-s\right)^{1.5}+\frac{2}{3}\left(\frac{2}{3}-s\right)^{1.5}\right]\right\} \\
& \times\left(\frac{1}{3} s+\frac{1}{4 \pi} \sin \left(2 \pi w_{n}(s)\right)\right. \\
& \left.+\frac{1}{18} z_{n}(t)+\frac{1}{5}\left(D_{t}^{0.3} w_{n}(t)\right)^{2}+D_{t}^{0.2} z_{n}(t)+\frac{10}{11}\right) d s \\
& +\frac{1}{\Gamma(2.5)} \int_{0}^{1} \frac{t^{1.5}}{1-l_{1} l_{2}}\left[\frac{2}{7 \Gamma(1.2)} \int_{0}^{\frac{1}{4}}\left(\frac{1}{4}-\tau\right)^{0.2} \tau^{1.5}(1-s)^{1.5} d \tau\right. \\
& -\frac{2}{7 \Gamma(1.2)} \int_{s}^{\frac{1}{4}}\left(\frac{1}{4}-\tau\right)^{0.2}(\tau-s)^{1.5} d \tau+\frac{3}{7 \Gamma(1.2)} \int_{0}^{\frac{3}{4}}\left(\frac{3}{4}-\tau\right)^{0.2} \tau^{1.5}(1-s)^{1.5} d \tau \\
& \left.-\frac{3}{7 \Gamma(1.2)} \int_{s}^{\frac{3}{4}}\left(\frac{3}{4}-\tau\right)^{0.2}(\tau-s)^{1.5} d \tau+\frac{1}{3} \cdot \frac{1}{3}^{1.5}(1-s)^{1.5}+\frac{2}{3} \cdot \frac{2}{3}^{1.5}(1-s)^{1.5}\right] \\
& \times\left(\frac{1}{2} s^{2}+\frac{1}{10} w_{n}(s)+\frac{1}{32} \sin \left(z_{n}(s)\right)+\frac{1}{50} D_{t}^{0.3} w_{n}(s)+\frac{1}{D_{t}^{0.2} z_{n}(s)-\frac{1}{2}}+2\right) d s \\
& -\frac{1}{\Gamma(2.5)} \int_{0}^{t} \frac{t^{1.5}}{1-l_{1} l_{2}}\left[\frac{1}{3}\left(\frac{1}{3}-s\right)^{1.5}+\frac{2}{3}\left(\frac{2}{3}-s\right)^{1.5}\right] \\
& \times\left(\frac{1}{2} s^{2}+\frac{1}{10} w_{n}(s)+\frac{1}{32} \sin \left(z_{n}(s)\right)\right. \\
& \left.+\frac{1}{50} D_{t}^{0.3} w_{n}(s)+\frac{1}{D_{t}^{0.2} z_{n}(s)-\frac{1}{2}}+2\right) d s \text {. }
\end{aligned}
$$

\section{Acknowledgements}

The authors would like to thank very much the anonymous referees for helpful comments and suggestions, which led to an improvement of presentation and quality of the work.

\section{Funding}

The work is supported by National Training Program of Innovation (Project No. 201910019172). The funding body plays an important role in the design of the study and analysis, calculation, and writing the manuscript.

\section{Competing interests}

The authors declare that they have no competing interests.

\section{Authors' contributions}

All authors contributed equally to the manuscript, read, and approved the final draft.

\section{Publisher's Note}

Springer Nature remains neutral with regard to jurisdictional claims in published maps and institutional affiliations.

Received: 7 April 2019 Accepted: 25 July 2019 Published online: 11 September 2019

\section{References}

1. Podlubny, I.: Fractional Differential Equations. Mathematics in Science and Engineering. Academic Press, New York (1999)

2. Kilbas, A.A., Srivastava, H.M., Trujillo, J.J.: Theory and Applications of Fractional Differential Equations. North-Holland Mathematics Studies. Elsevier, Amsterdam (2006)

3. Liu, Y., Ahmad, B.: A study of impulsive multiterm fractional differential equations with single and mutiple base point and applications. Sci. World J. 2014, Article ID 194346 (2014)

4. Hilfer, R.: Applications of Fractional Calculus in Physics. World Scientific, Singapore (2000)

5. Zhang, W., Bai, Z., Sun, S.: Extremal solutions for some periodic fractional differential equations. Adv. Differ. Equ. 2016 $179(2016)$

6. Liu, X., Jia, M., Ge, W.: The method of lower and upper solutions for mixed fractional four-point boundary value problem with $p$-Laplacian operator. Appl. Math. Lett. 65, 56-62 (2017) 
7. Yan, F., Zuo, M., Hao, X.: Positive solution for a fractional singular boundary value problem with $p$-Laplacian operator. Bound. Value Probl. 2018(1), 51 (2018)

8. Xu, M., Han, Z.: Positive solutions for integral boundary value problem of two-term fractional differential equations. Bound. Value Probl. 2018(1), 100 (2018)

9. Jia, M., Liu, X:: Multiplicity of solutions for integral boundary value problems of fractional differential equations with upper and lower solutions. Appl. Math. Comput. 232, 313-323 (2014)

10. Ahmed, H.M., El-Borai, M.M.: Hilfer fractional stochastic integro-differential equations. Appl. Math. Comput. 331, 182-189 (2018)

11. Sheng, K., Zhang, W., Bai, Z:: Positive solutions to fractional boundary-value problems with p-Laplacian on time scales. Bound. Value Probl. 2018(1), 70 (2018)

12. Jiang, J., Liu, W., Wang, H.: Positive solutions to singular Dirichlet-type boundary value problems of nonlinear fractional differential equations. Adv. Differ. Equ. 2018(1), 169 (2018)

13. Ahmad, B., Ntouyas, S.K., Alsaedi, A.: Existence of solutions for fractional differential equations with nonlocal and average type integral boundary conditions. J. Appl. Math. Comput. 53, 129-145 (2017)

14. Bo, S.: Monotone iterative technique and positive solutions to a third-order differential equation with advanced arguments and Stieltjes integral boundary conditions. Adv. Differ. Equ. 2018(1), 218 (2018)

15. Guo, L., Liu, L., Wu, Y.: Uniqueness of iterative positive solutions for the singular fractional differential equations with integral boundary conditions. Bound. Value Probl. 2016, 147 (2016)

16. Wang, G., Pei, K., Agarwal, R.P., Zhang, L., Ahmad, B.: Nonlocal Hadamard fractional boundary value problem with Hadamard integral and discrete boundary conditions on a half-line. J. Comput. Appl. Math. 343, 230-239 (2018)

17. Song, Q., Bai, Z.: Positive solutions of fractional differential equations involving the Riemann-Stieltjes integral boundary condition. Adv. Differ. Equ. 2018(1), 183 (2018)

18. Liu, X., Jia, M.: Solvability and numerical simulations for BVPs of fractional coupled systems involving left and right fractional derivatives. Appl. Math. Comput. 353, 230-242 (2019)

19. Liu, X., Jia, M.: The method of lower and upper solutions for the general boundary value problems of fractional differential equations with $p$-Laplacian. Adv. Differ. Equ. 2018, 28 (2018)

20. Ali, N., Shah, K., Baleanu, D., Arif, M., Khan, R.A.: Study of a class of arbitrary order differential equations by a coincidence degree method. Bound. Value Probl. 2017(1), 111 (2017)

21. Bai, Z., Zhang, Y.: Solvability of fractional three-point boundary value problems with nonlinear growth. Appl. Math. Comput. 218(5), 1719-1725 (2011)

22. Zhang, Y., Bai, Z., Feng, T.: Existence results for a coupled system of nonlinear fractional three-point boundary value problems at resonance. Comput. Math. Appl. 61(4), 1032-1047 (2018)

23. Alsaedi, A., Ntouyas, S.K., Garout, D., Ahmad, B.: Coupled fractional-order systems with nonlocal coupled integral and discrete boundary conditions. Bull. Malays. Math. Sci. Soc. 42, 241-266 (2017)

24. Dimplekumar, C., Avadhesh, K.: Existence, uniqueness and Ulam's stability of solutions for a coupled system of fractional differential equations with integral boundary conditions. Mathematics 6(6), 96 (2018)

25. Cui, M., Zhu, Y., Pang, H.: Existence and uniqueness results for a coupled fractional order systems with the multi-strip and multi-point mixed boundary conditions. Adv. Differ. Equ. 2017(1), 224 (2017)

26. Yang, W.: Monotone iterative technique for a coupled system of nonlinear Hadamard fractional differential equations. J. Appl. Math. Comput. (2018). https://doi.org/10.1007/s12190-018-1192-x

27. Wen, R., Zhao, P.: A medium-shifted splitting iteration method for a diagonal-plus-Toeplitz linear system from spatial fractional Schrödinger equations. Bound. Value Probl. 2018(1), 45 (2018)

28. Li, Y., Shah, K., Khan, R.A.: Iterative technique for coupled integral boundary value problem of non-integer order differential equations. Adv. Differ. Equ. 2017(1), 251 (2017)

\section{Submit your manuscript to a SpringerOpen ${ }^{\circ}$ journal and benefit from:}

- Convenient online submission

- Rigorous peer review

- Open access: articles freely available online

- High visibility within the field

- Retaining the copyright to your article

Submit your next manuscript at $\gg$ springeropen.com 